\title{
Analysis of superframe duration adjustment scheme for IEEE 802.15.4 networks
}

\author{
Bih-Hwang Lee ${ }^{1 *}$, Eppy Yundra ${ }^{1}$, Huai-Kuei Wu ${ }^{2}$ and M Udin Harun Al Rasyid ${ }^{3}$
}

\begin{abstract}
The challenge of the IEEE 802.15 .4 beacon-enabled mode is how to improve throughput and bandwidth utilization in contention access period (CAP) and contention free period (CFP), respectively. This article proposes a scheme to improve IEEE 802.15.4 medium access control, called superframe duration adjustment scheme (SUDAS), which analyzes the overall of the IEEE 802.15.4 not only CAP but also CFP. SUDAS is expected to effectively allocate guaranteed time slot to the requested devices, it adjusts the length of the slot in superframe duration based on the length of the packet data. This article also presents a comprehensive Markov chain analysis for SUDAS, especially for star topology, to predict the probability of successful transmission, network goodput, average bandwidth utilization, as well as total network energy consumption. The validity of the analytical model is proven by closely matching the simulation experiments. SUDAS performs better than other algorithms in terms of the probability of successful packet transmission, network goodput, average bandwidth utilization, and total energy consumption.
\end{abstract}

Keywords: IEEE 802.15.4; Markov chain; Guaranteed time slot; Energy consumption

\section{Introduction}

The IEEE 802.15.4 standard has been designed to specify the physical layer (PHY) and medium access control (MAC) sublayer for low power consumption, short transmission range, and low-rate wireless personal area network (LR-WPAN) [1]. IEEE 802.15.4 can operate on beacon- and non-beacon-enabled modes and has three kinds of topologies: star, peer-to-peer, and cluster tree topologies. The network coordinator transmits beacon to synchronize and provide necessary information to the devices in beacon enabled-mode, while the unslotted carrier sense multiple access with collision avoidance (CSMA/ CA) protocol is used in non-beacon-enabled mode. The IEEE 802.15.4 MAC supports not only contention-based mechanism in contention access period (CAP) but also guaranteed time slot (GTS) in contention free period (CFP) scheme under beacon-enabled-mode. The GTS transmission in CFP can avoid packet drop due to collisions in the contention based protocol (i.e., CSMA/CA in CAP). The limited number of allowable retransmissions and the number of backoffs as specified in the standard

\footnotetext{
* Correspondence: bhlee@mail.ntust.edu.tw

${ }^{1}$ National Taiwan University of Science and Technology, 43, Keelung Rd, Section 4, Taipei 106, Taiwan

Full list of author information is available at the end of the article
}

can reduce energy consumption caused by carrier sensing. However, the performance of CAP and CFP are related each other because the number of request GTS packets successfully received by network coordinator may decrease if the contention level in CAP increases, which will decrease the throughput of CFP and vice versa. The problem in part of CFP in terms of GTS mechanism is how the network coordinator allocates time slot duration for the device nodes which request GTS. However, if the allocated GTS slot for device node is inappropriate or less than the available bandwidth, the wasted bandwidth will increase which degrade the performance of network.

The performance analysis of IEEE 802.15.4 MAC is one of the important research topics in wireless sensor network. That means research overall performance of the IEEE 802.15.4 MAC includes CAP and CFP simultaneously still prepossess. The challenge of the IEEE 802.15.4 beacon-enabled mode is how to improve throughput and bandwidth utilization in CAP and CFP, respectively. The authors of [2] present an evaluation of the slotted CSMA/ CA of IEEE 802.15.4 based on all of its frequency, which only analyzes each frequency and compares with each other but not to propose a method. In [3], the authors analyze the performance of IEEE 802.15.4 MAC by using node state and channel state models that are simple but 
accurate. The authors also present an analytical model for the slotted CSMA/CA algorithm adopted in the CAP of the beacon-enabled mode in IEEE 802.15.4 MAC, which only considers for the saturated mode but not for acknowledgement (ACK).

Several mathematical analyses based on Markov chain models have been proposed to analyze the performance of IEEE 802.15.4, but they do not consider packet retransmissions [4-10]. Some of the modified Markov chain models have been investigated by considering packet retransmissions but not considering the defer transmission [11-14]. In [15-18], the authors propose the Markov chain models with considering the postpone transmission. In [19], the analytical model based on Markov chain for multi-hop cluster network has been studied without considering ACK to confirm the successful of data packet transmission. However, all of the abovementioned models only consider for the contention-based transmission, i.e., only for CAP.

The authors of [20] propose a methodology to analyze the GTS mechanism in CFP. In [21], the authors propose an analytical model based on Markov chain for GTS allocation mechanism in CFP. In [22], the authors provide an analysis for channel access during CAP and CFP. However, the purpose of the CFP transmission is to retransmit the packet that is not successful transmitted in CAP to cope with hidden node collisions. A number of mechanisms have been proposed to gain effective GTS allocation. Multi-beacon superframe (MBS) and greedy GTS allocation (GGA) algorithms are proposed to decompose a single beacon interval into multiple subbeacon intervals in order to reduce the bandwidth waste problem [23]. However, the increasing number of beacon transmission in MBS and GGA may increase the energy consumption. A delay-bound analysis for an implicit GTS allocation is proposed to analyze the impact on the bandwidth utilization and delay by using numerical network calculus analysis [24]. The authors of [25] analyze the priorities of devices to determine for GTS allocation, while the authors of [26] further propose an adaptive GTS allocation scheme (AGA) using two phases to assign the priorities of devices and schedule GTS. In [27], the authors propose a method for GTS allocation with improved bandwidth utilization, known as a new GTS allocation scheme with bandwidth utilization (ANBU), which allows more devices to share the bandwidth within the same period. However, CFP is always divided into 16 equal-length slots without considering the value of superframe order (SO) and the arrival rate of data packets; therefore the length of each slot increases if the SO value increases, which causes waste bandwidth. In [28], the authors propose an optimization-based GTS allocation scheme designed according to the priorities of the devices and knapsack problem. The network coordinator collects the bandwidth requests from devices, then allocates GTS to the demanded devices by using fractional knapsack problem given by their priorities. In [29], the authors present a new GTS allocation scheme (NGAS) for IEEE 802.15.4, which divides the CFP into 32 equal-sized slots. NGAS did not consider the value of $\mathrm{SO}$ and the arrival rate of data packets, so that it may cause inefficient bandwidth if the value of $\mathrm{SO}$ or arrival rate increases. The authors of [30] propose a dynamic CFP allocation and opportunity contention-based protocol to request CFP slots for devices in wireless body area network (WBAN) environment. The length of CFP allocation period may increase as the number of requested CFP slots increases, but the length of CAP used by the devices will be decreased. However, all of the aforementioned models only consider GTS transmission, i.e., only for CFP. In other words, they did not consider the overall performance for both CAP and CFP.

This article proposes a superframe duration adjustment scheme (SUDAS) for IEEE 802.15.4 which is the extended work from [18] and [31]. In [18], the authors focus on how to decrease the collisions between beacons or even between beacon and data packets by adjusting the beacon starting times of PAN and coordinator nodes for cluster tree topology, which only considers for the part of CAP but not for GTS slot allocation in the corresponding Markov chain model. SUDAS focuses on assigning adjustable length of GTS slot based on the length of packet and also deciding the precise time for the GTS starting time (GTSstart) and the GTS length (GTSlength) for star topology. SUDAS is expected to effectively allocate GTS to the requested device nodes to improve bandwidth utilization in CFP, while the length of CAP can be used by other device nodes to transmit their data packets which do not receive the allocated GTS. The performance of SUDAS for star topology is analyzed by the Markov chain model modified from [18] for considering packet retransmission, ACK, defer transmission and GTS allocation, which is to obtain the probability of success transmissions, network goodput, energy consumption, and average bandwidth utilization for IEEE 802.15.4 MAC.

\section{Overview of IEEE 802.15.4}

The IEEE 802.15.4 standard can operate in beaconenabled mode and non-beacon-enabled mode. In beaconenabled mode, each node employs two system parameters: beacon order $(\mathrm{BO})$ and $\mathrm{SO}$. The parameter $\mathrm{BO}$ decides the length of beacon interval (BI), where $\mathrm{BI}=$ aBaseSuperframeDuration $\times 2^{\mathrm{BO}}$ symbols and $0 \leq \mathrm{BO} \leq 14$; while the parameter $\mathrm{SO}$ decides the length of superframe duration (SD), where $\mathrm{SD}=$ aBaseSuperframeDuration $\times 2^{\mathrm{SO}}$ symbols and $0 \leq \mathrm{SO} \leq \mathrm{BO} \leq 14$. The value of aBaseSuperframeDuration is fixed to 960 symbols. The format of the superframe is defined by the network coordinator as 
shown in Figure 1. Furthermore, the active portion of each superframe consists of three parts: beacon, CAP, and CFP, which is divided into 16 equal length slots. The length of one slot is equal to aBaseSlotDuration $\times 2^{\mathrm{SO}}$ symbols, where aBaseSlotDuration is equal to 60 symbols.

In CAP, each node performs the CSMA/CA algorithm before transmitting data packet or control frame. Each node maintains three parameters: the number of backoffs (NB), contention window (CW), and backoff exponent $(\mathrm{BE})$. The initial values of $\mathrm{NB}, \mathrm{CW}$, and $\mathrm{BE}$ are equal to 0,2 , and macMinBE, respectively, where mac$M i n B E$ is equal to 3 . In the located boundary of the next backoff period, a node takes delay for random backoff between 0 and $2^{\mathrm{BE}}-1\left(2^{\mathrm{BE}}\right.$ minus 1$)$ unit backoff period (UBP), where UBP is equal to 20 symbols (or 80 bits). A node performs clear channel assessment (CCA) to make sure whether the channel is idle or busy, when the number of random backoff periods is decreased to 0 . The value of $\mathrm{CW}$ will be decreased by one if the channel is idle; and the second CCA will be performed if the value of $\mathrm{CW}$ is not equal to 0 . If the value of $\mathrm{CW}$ is equal to 0 , it means that the channel is idle after twice CCA; then a node is committed the data transmission. However, if the CCA is busy, the value of $\mathrm{CW}$ will be reset to 2; the value of $\mathrm{NB}$ is increased by 1 ; and the value of $\mathrm{BE}$ is increased by 1 up to the maximum $\mathrm{BE}$ (macMaxBE), where the value $\operatorname{macMaxBE}$ is equal to 5 . The node will repeatedly take random delay if the value of NB is less than the value of macMaxCSMABackoff, where the value of macMaxCSMABackoff is equal to 4; and the transmission attempt fails if the value of $\mathrm{NB}$ is greater than the value of macMaxCSMABackoff.

On the other hand, to transmit packet in CFP, a node has to request the usage of GTS by sending GTS request packet to network coordinator in the CAP of the previous superframe. The network coordinator allocates GTS to the device node if it successfully receives the request packet, then the device node transmits its packets by using the allocated GTS without contention.

\section{The description of SUDAS}

A star topology consists of one network coordinator and several device nodes, while the network coordinator periodically sends beacon frames to the device nodes. The network coordinator allocates the dedicated slots

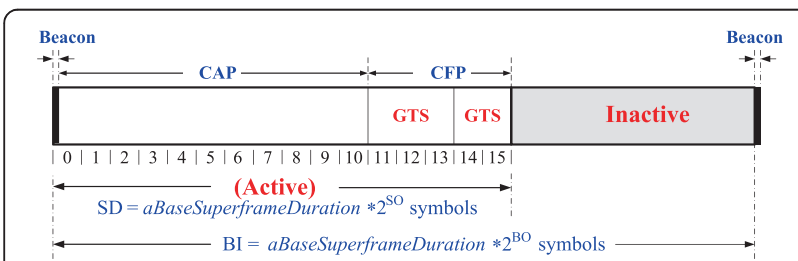

Figure 1 An example of superframe structure. for its device nodes, if it receives the requests for GTS packets in the CAP period, otherwise, the device nodes shall transmit their packets with contention in CAP. This article proposes a superframe duration adjustment scheme (SUDAS), to analyze both CAP and CFP for IEEE 802.15.4 MAC. SUDAS aims to accurately decide the values of GTS slot based on the length of packet size and packet arrival rate. SUDAS mainly improves the probability of success transmission, network goodput, average bandwidth utilization, and energy consumption by managing the GTS allocation for the requested device nodes. SUDAS can be expanded by considering the length of data packet, the SO value, and packet arrival rate. According to the IEEE 802.15.4 standard, let us denote $T_{\mathrm{sd}}$ to be the time of SD as shown in Equation 1, where aBaseSuperframeDuration and $R_{\mathrm{s}}$ are the minimum duration of a superframe and data symbol rate with the values of $960 \mathrm{symbols}$ and $62,500 \mathrm{symbol} / \mathrm{s}$, respectively. Let us also denote $T_{\text {slot }}$ as the time of one slot duration, which can be obtained by Equation 2 .

$$
\begin{gathered}
\left.T_{\text {sd }}=\frac{\text { aBaseSuperframeDuration } \times 2^{\mathrm{SO}}}{R \mathrm{~s}} \text { [in seconds }\right] \\
T_{\text {slot }}=\frac{T_{\text {sd }}}{16}[\text { in seconds }]
\end{gathered}
$$

Each device node with an allocated GTS ensures that the data transmission time, waiting time for ACK, time to transmit ACK packet, and interframe spacing (IFS) duration can be completed before the end of its GTS period. Let us denote $T_{f}$ be the time to transmit one data packet and receive ACK packet, which can be obtained by Equation 3, where $T_{\text {data }}, T_{\text {Lack }}, T_{\text {ack }}$, and $T_{\text {LIFS }}$ are the time to transmit data packet, time to waiting for ACK packet, time to transmit ACK packet, and time duration of IFS, respectively. The length of $L_{\text {ack }}$ is equal to 88 bits, whereas the length of $L_{\mathrm{IFS}}$ is equal to macMinLIFSPeriod (160 bits) if the length of packet is greater than aMaxSIFSFrameSize (144 bits), otherwise, it is equal to macMinSIFSPeriod (48 bits).

$$
T_{\mathrm{f}}=T_{\text {data }}+T_{\text {Lack }}+T_{\text {ack }}+T_{\text {LIFS }}
$$

There are two types of packets, time-critical and nontime-critical packets, to be transmitted from nodes to the coordinator. In CAP, the packet transmission delay cannot be guaranteed, because packets are transmitted by using the CSMA/CA algorithm. Conversely, the packet transmission delay can be guaranteed in CFP, because packets are transmitted by using the allocated GTS without contention. In this article, we assume that each node generates time-critical and non-time-critical packets with probabilities $P$ and $(1-P)$, respectively. Let us denote $T \mathrm{x}_{\mathrm{n}}$ be the time to transmit a data packet 
according to its arrival rate by device $\mathrm{n}$ as shown in Equation 4, where $\lambda_{\mathrm{n}}$ and $N_{\mathrm{GTS}}$ are the arrival rate of data packets at device $n$ and the number of nodes to be allocated GTS slots in CFP, respectively.

$$
T x_{n}=\frac{\lambda_{\mathrm{n}} \times T_{\mathrm{sd}} \times P}{R_{\mathrm{b}}}, \mathrm{n} \in\left(1, N_{\mathrm{GTS}}\right)
$$

According to IEEE 802.15.4 standard, the maximum value to allocate the GTS slot duration is seven. Let us denote adjslot be the integer value that will be used as the adjustment for the $T_{\text {slot }}$ of IEEE 802.15.4 standard become new smaller adjustment time of one slot duration. The value adjslot can be calculated by Equation 5 . Let us denote $T_{\text {sudas }}$ be the new time of one slot duration in SUDAS, which can be calculated by Equation 6. Let us denote Nsudasslot $t_{n}$ be the number of request slots for each GTS of SUDAS by device $n$, which is calculated by Equation 7. Let us denote NumSuperframeSlot be the number of slots in a superframe duration, which is equal to 16 according to IEEE 802.15.4 standard. Let us denote GTSstar $_{\mathrm{n}}$, and GTSlength $\mathrm{n}$ as the starting time, and the length of a GTS allocation for device $n$, which can be calculated by Equations 8 to 9, respectively. Let us also denote $\mathrm{CAP}_{\text {sudasslot }}$ and $\mathrm{CAP}_{\text {sudaslength }}$ be the number of CAP slots and the time of CAP period based on SUDAS, which can be obtained by Equations 10 and 11, respectively, where $L_{\text {beacon }}$ is the length of beacon in bits while $T_{\text {beacon }}$ is the time interval of beacon.

$$
\text { adjslot }=\left\lfloor\frac{T_{\text {slot }}}{T_{\mathrm{f}}}\right\rfloor, \text { where }\lfloor x\rfloor \text { is the maximum integer }
$$

but less than $x$.

$T_{\text {sudas }}=\frac{T_{\text {slot }}}{\text { adjslot }}$

Nsudasslot $_{\mathrm{n}}=\left\lceil\frac{T x_{n}}{T_{\text {sudas }}}\right\rceil$, where $\lceil x\rceil$ is the minimum integer but greater than $x$.

$$
\begin{aligned}
& \text { GTSstart }_{\mathrm{n}}= \text { NumSuperframeSlot } \times T_{\text {slot }}-T_{\text {sudas }} \\
& \times \sum_{i=1}^{n} \text { Nsudasslot }_{\mathrm{i}}, \quad 1 \leq n \leq N_{\mathrm{GTS}} \\
& \text { GTSlength }_{\mathrm{n}}=\text { Nsudasslot }_{\mathrm{n}} \times T_{\text {sudas }} \\
& \text { CAP }_{\text {sudasslot }}= \text { NumSuperframeSlot }-\left[\sum_{n=1}^{N_{\mathrm{GTS}}} \frac{\text { Nsudasslot }_{\mathrm{n}}}{\text { adjslot }}\right]
\end{aligned}
$$

$$
\begin{aligned}
\mathrm{CAP}_{\text {sudaslength }}= & \text { NumSuperframeSlot } \\
& \times T_{\text {slot }}-T_{\text {beacon }}-T_{\text {sudas }} \\
& \times \sum_{n=1}^{N_{\mathrm{GTS}}} \text { Nsudasslot }_{\mathrm{n}}
\end{aligned}
$$

For more details about the aforementioned description, we can explain SUDAS with flowchart as shown in Figure 2. We consider a star topology network having one network coordinator and several device nodes with the same value of six for $\mathrm{SO}$ and BO. By using Equation 5 , we get the value of adjslot to be 16. Based on the IEEE 802.15.4 standard, if each node sends to request one slot GTS in IEEE 802.15.4, and its request successfully received by network coordinator, thus the number of slots needed for CFP is seven as shown in Figure 3. In SUDAS, the number of slots needed for CFP is not more than one in the 15th slot. Furthermore, if there are more than seven device nodes in the star topology, the device nodes which are not allocated GTS can transmits their data packets more in CAP period because the CAP duration of SUDAS $\left(\mathrm{CAP}_{\text {sudaslength }}\right)$ is increased as shown in Figure 4. By using SUDAS, the CFP period in superframe duration effectively increases the average bandwidth utilization. On the other hand, the device nodes which are not getting GTS allocation can increase their data packets transmitted in CAP because the CAP duration is increased.

\section{Analysis of SUDAS}

In this section, the proposed SUDAS based on the IEEE 802.15.4 MAC use slotted carrier sense multiple access with collision avoidance (CSMA/CA) for part of CAP and GTS transmission for part of CFP. This article also taking into account the case of acknowledged uplink data transmission investigated comprehensively via Markov chain model as shown in Figure 5 . Let $b_{\mathrm{i}, \mathrm{j}, \mathrm{k}}$ be the stationary probability at the stochastic state $(s(t)=i, c(t)=j$, and $r(t)=k)$, where $s(t)$, $c(t)$, and $r(t)$ represent backoff stage, backoff counter, and number of retransmissions, respectively, shown as Equation 12, where $b_{\mathrm{i},-1, \mathrm{k}}, b_{\mathrm{i},-2, \mathrm{k}}$ and $b_{\mathrm{i},-3, \mathrm{k}}$ are the stationary probabilities for the first $\mathrm{CCA}\left(\mathrm{CCA}_{1}\right)$, the second CCA $\left(\mathrm{CCA}_{2}\right)$, and packet transmission, respectively, at the $i$ th backoff stage and the $k$ th retransmission. Let $b_{\mathrm{Si}, \mathrm{k}}$ and $b_{\mathrm{Ci}, \mathrm{k}}$ be the stationary probabilities of the successful transmission and collision at the states of $S_{\mathrm{i}, \mathrm{k}}$ and $C_{\mathrm{i}, \mathrm{k}}$ as shown in Equations 13 and 14, respectively, where $m$ and $R$ are the maximum NB stage and retransmissions, i.e., they are equal to 4 and 3 , respectively. Let $b_{\mathrm{SGi}, \mathrm{k}}$ and $b_{\mathrm{Di}, \mathrm{k}}$ be the stationary probabilities of the successful request for GTS packets and deferred transmission at the states of $\mathrm{SG}_{\mathrm{i}, \mathrm{k}}$ and $\mathrm{D}_{\mathrm{i}, \mathrm{k}}$ for the $i$ th backoff 


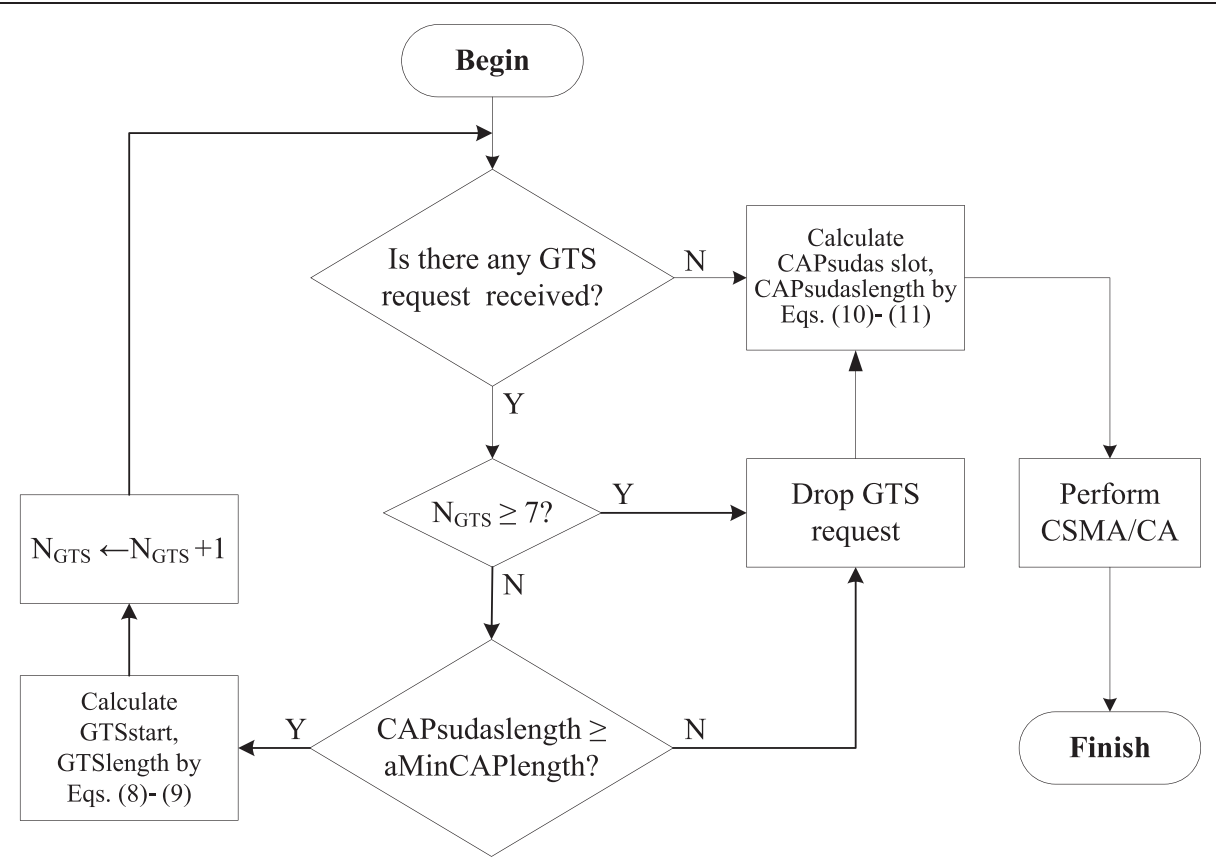

Figure 2 The flowchart of SUDAS.

stage and the $k$ th retransmission as shown in Equations 15 and 16 , respectively.

$$
\begin{aligned}
& b_{i, j, k}=\lim _{t \rightarrow \infty} P\{s(t)=i, c(t)=j, r(t)=k\}, \text { for } i \in(0, m), \\
& j \in\left(-3, w_{i}-1\right), \quad k \in(0, R) \\
& b_{S_{i, k}}=\lim _{t \rightarrow \infty} P\left\{S_{s(t)}=S_{i}, \quad r(t)=k\right\}, \quad i \in(0, m), \quad k \in(0, R) \\
& b_{C_{i, k}}=\lim _{t \rightarrow \infty} P\left\{C_{s(t)}=C_{i}, \quad r(t)=k\right\}, \quad i \in(0, m), \quad k \in(0, R) \\
& b_{\mathrm{SG}_{i, k}}=\lim _{t \rightarrow \infty} P\left\{\mathrm{SG}_{s(t)}=\mathrm{SG}_{i}, \quad r(t)=k\right\}, \quad i \in(0, m), \quad k \in(0, R) \\
& b_{D_{i, k}}=\lim _{t \rightarrow \infty} P\left\{D_{s(t)}=D_{i}, \quad r(t)=k\right\}, \quad i \in(0, m), \quad k \in(0, R)
\end{aligned}
$$

$$
\begin{array}{|c|c|c|c|c|c|c|c|c|c|c|c|c|c|c|c|c|}
\multicolumn{10}{|c|}{\text { CFPstdlength }} \\
\hline 0 & 1 & 2 & 3 & 4 & 5 & 6 & 7 & 8 & 9 & 10 & 11 & 12 & 13 & 14 & 15 \\
\hline
\end{array}
$$

Figure 3 An example of GTS allocation in IEEE 802.15.4.
Let us explain the parameters used in the Markov chain model as follows. Let $w_{i}=2^{\mathrm{BE}_{i}}$ be the backoff window at the $i$ th backoff stage of a device, where the backoff exponent $\mathrm{BE}_{\mathrm{i}}=3,4,5,5$, and 5 for $0 \leq i \leq m$. An IDLE state means that a device node has no packet to transmit. Let us denote $q$ be the probability that a packet arrives at a node during the active period.

The MAC sublayer should transmit its packet if the remaining CSMA/CA steps, i.e., CCA analyses, the frame transmission, and any ACK can be completed before the end of $\mathrm{CAP}_{\text {sudaslength }}$. Conversely, if the current $\mathrm{CAP}_{\text {sudaslength }}$ has not enough slots to transmit data packets, it should defer transmission until the beginning of the $\mathrm{CAP}_{\text {sudaslength }}$ in the next superframe duration. Let $d$ be the probability of defer transmission that no enough slot is left in the current $\mathrm{CAP}_{\text {sudaslength }}$ to transmit data packet, which can be obtained by Equation 17, where $T_{\mathrm{txCCA}}$ is the time to transmit CCA.

$$
d=\frac{2 T_{t x \mathrm{CCA}}+T_{f}}{\mathrm{CAP}_{\text {sudaslength }}}
$$

Let us denote $\alpha$ and $\beta$ be the probabilities that $\mathrm{CCA}_{1}$ and $\mathrm{CCA}_{2}$ are busy, respectively. $\mathrm{CCA}_{1}$ busy means that the device node at one of the $\mathrm{CCA}_{1}$ states while at least one of the other nodes at packet transmission state, while $\mathrm{CCA}_{2}$ busy means that the device node at one of the $\mathrm{CCA}_{2}$ states while at least one of the other nodes at packet transmission state. Let us also denote $P_{\text {coll }}$ to be the probability of the collision of packet transmission 


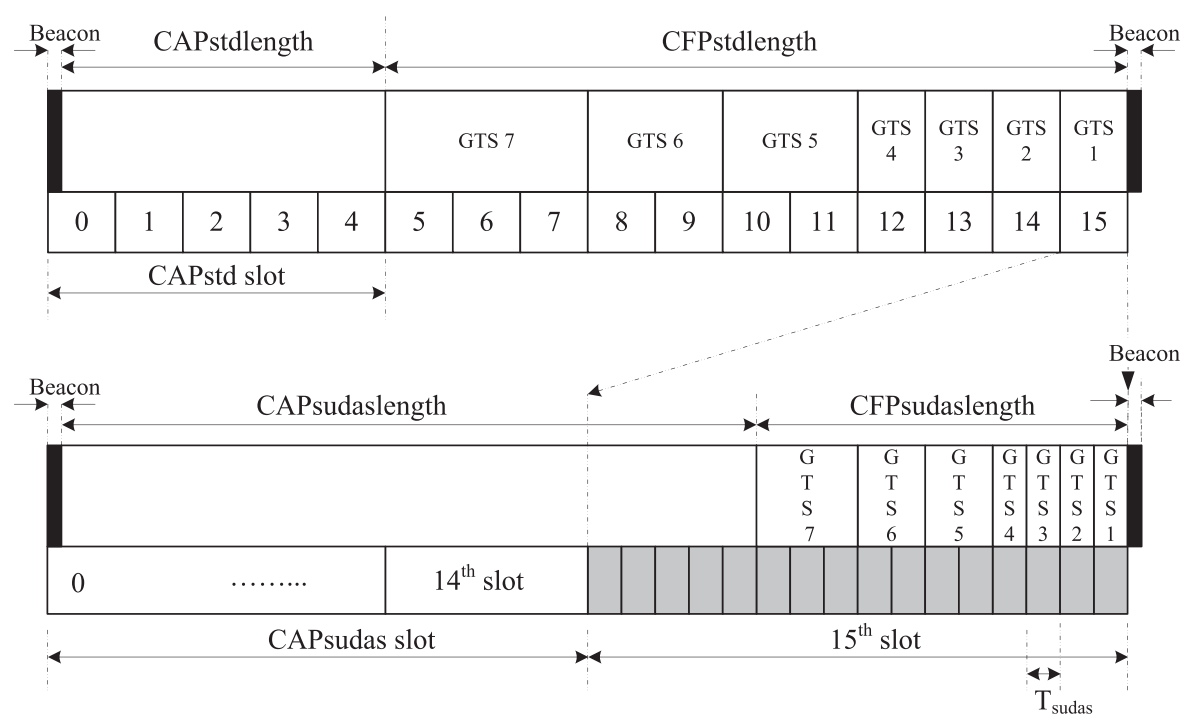

Figure 4 An example of GTS allocation in SUDAS.

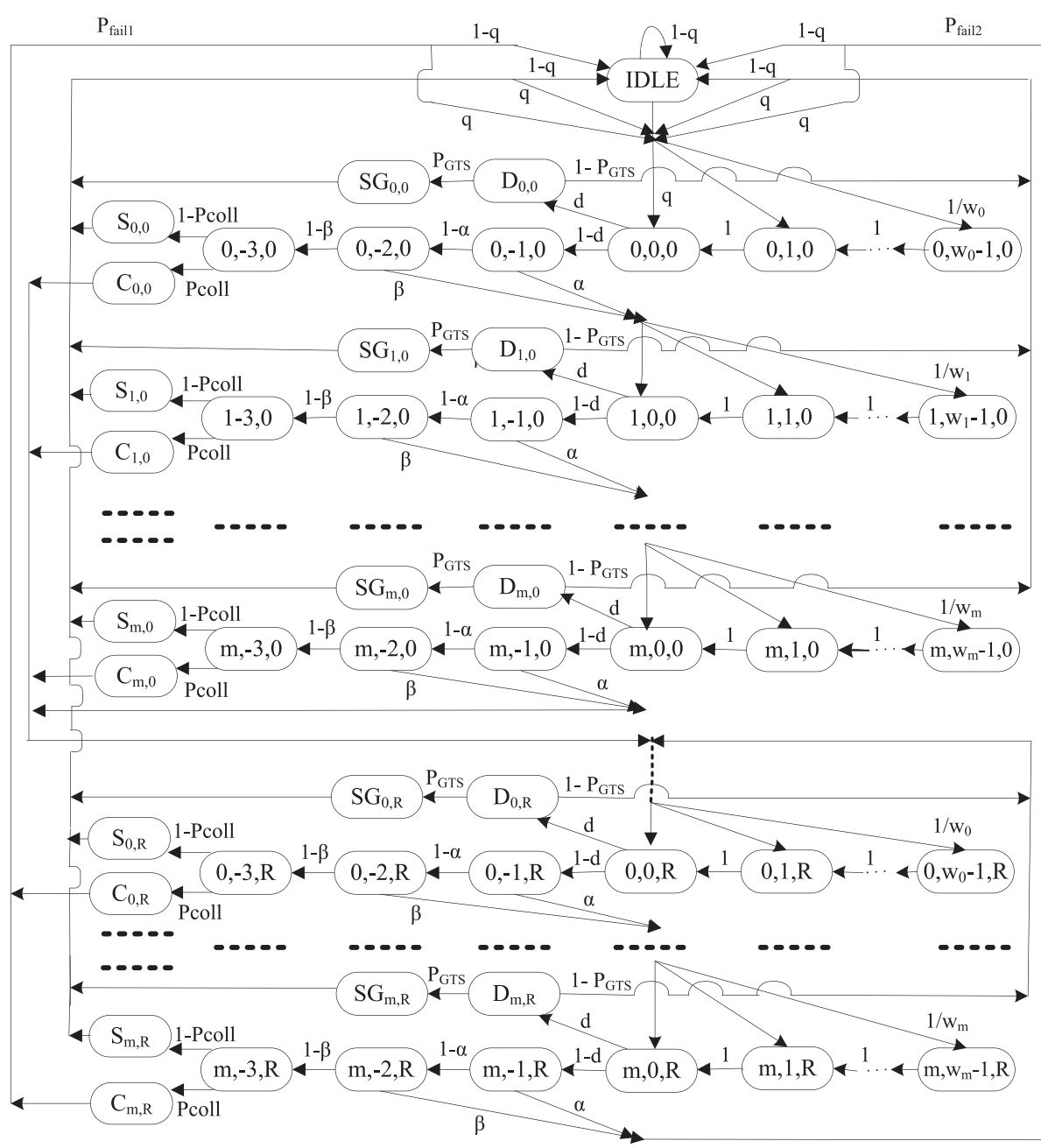

Figure 5 Markov chain model for SUDAS. 
after $\mathrm{CCA}_{2}$, i.e., the device node at packet transmission state while at least one of the other nodes in the packet transmission state at the same time. Let us also denote $P_{\text {fail1 }}$ and $P_{\text {fail } 2}$ to be the probabilities of fail transmission due to the maximum number of retransmissions after collisions and no channel to use after reaching the maximum backoff stage at the maximum retransmission stage, respectively.

To analyze the Markov chain model, several state transition probabilities are evaluated as shown from Equations 18 to 26 . Equation 18 states the probability that the backoff counter is decreased after each slot. Equation 19 gives the probability of finding busy channel either in $\mathrm{CCA}_{1}$ and $\mathrm{CCA}_{2}$. Equation 20 states the probability of picking a backoff state in the next retransmission stage after the collision of packet transmission when having enough time to send packet in the remaining active period and channel idle in both $\mathrm{CCA}_{1}$ and $\mathrm{CCA}_{2}$. Equation 21 states the probability of entering the IDLE state after the collision of packet transmission while reaching the maximum retransmission stage after finding the remaining active period to be enough to send packet and channel idle in both $\mathrm{CCA}_{1}$ and $\mathrm{CCA}_{2}$. Equation 22 states the probability that the remaining $\mathrm{CAP}$ is not enough to send packet and need to defer and then pick the backoff state in the next superframe. Equation 23 states the probability of successful packet transmission and picking new random backoff at the first backoff stage. Equation 24 states the probability of entering the IDLE state if the node has no data packet to transmit after successful packet transmission. Equation 25 states the probability of entering the IDLE state due to channel access failure. Equation 26 states the probability of going to the first backoff stage from the IDLE state if the node has data packet to transmit.

$$
P(i, j, k \mid i, j+1, k)=1, i \in(0, m), j \in\left(0, w_{i}-2\right), k \in(0, R)
$$

$$
\begin{aligned}
P(i, j, k \mid i-1,0, k)= & \frac{w_{i}-j}{w_{i}}(1-d)(\alpha)+(1-d)(1-\alpha) \beta \\
= & \frac{w_{i}-j}{w_{i}}(1-d)[\alpha+(1-\alpha) \beta], \\
& i \in(1, m), j \in\left(0, w_{i}-1\right), k \in(0, R)
\end{aligned}
$$

$P(0,0, k \mid i, 0, k-1)=(1-d)(1-\alpha)(1-\beta)\left(P_{\text {coll }}\right), \quad k \in(1, R)$

$$
\begin{aligned}
& P(\operatorname{IDLE} \mid i, 0, R)=(1-d)(1-\alpha)(1-\beta)\left(P_{\text {coll }}\right)(1-q) \quad(21) \\
& P(0, j, k \mid i, 0, k)= \begin{cases}\frac{w_{0}-j}{w_{0}}(d)\left(1-P_{\mathrm{GTS}}\right) \quad q, & \text { for } k=0 \\
\frac{w_{0}-j}{w_{0}}(d)\left(1-P_{\mathrm{GTS}}\right), & \text { for } k \in(1, R)\end{cases}
\end{aligned}
$$

$$
\begin{aligned}
P(0, j, 0 \mid i, 0, k)= & \frac{w_{0}-j}{w_{0}}(1-\mathrm{d})(1-\alpha)(1-\beta)\left(1-P_{\text {coll }}\right) q, \\
& i \in(0, m), j \in\left(0, w_{i}-1\right), k \in(0, R)
\end{aligned}
$$

$P(\operatorname{IDLE} \mid i, 0, k)=(1-d)(1-\alpha)(1-\beta)\left(1-P_{\text {coll }}\right)(1-q)$,

$$
i \in(0, m), k \in(0, R)
$$

$$
\begin{aligned}
& P(\operatorname{IDLE} \mid m, 0, R)=(1-d)[\alpha+(1-\alpha)(\beta)](1-q) \\
& P(0, j, 0 \mid \mathrm{IDLE})=\frac{w_{0}-j}{w_{0}} q, j \in\left(0, w_{i}-1\right)
\end{aligned}
$$

By using Equation 19, the stationary probability $b_{\mathrm{i}, \mathrm{j}, \mathrm{k}}$ can be obtained by Equation 27. From Equation 20, $b_{0,0, \mathrm{k}}$ can be obtained by Equation 28, where $Y$ and $X$ are the probabilities of entering the next backoff stage and the collision of packet transmission in a certain backoff stage, respectively. Similarly, $b_{\mathrm{i}, 0, \mathrm{k}}$ can be obtained by Equation 29 . Finally, the steady-state probabilities to perform random backoff $\left(P_{\text {randb }}\right), \mathrm{CCA}_{1}\left(P_{\mathrm{CCA} 1}\right), \mathrm{CCA}_{2}\left(P_{\mathrm{CCA} 2}\right)$, packet transmission $\left(P_{\mathrm{pt}}\right)$, successful packet transmission $\left(P_{\text {suc }}\right)$, collision of packet transmission $\left(P_{\text {copt }}\right)$, deferred transmission $\left(P_{\mathrm{dtx}}\right)$, successful request for GTS packet $\left(P_{\mathrm{sg}}\right)$, and idle state $\left(P_{\text {idle }}\right)$ can be obtained from Equations 30 to 38 , respectively, where $P_{\mathrm{GTS}}$ is the probability of requesting GTS allocation. Since the sum of probabilities in the Markov chain must be equal to one, we have Equation 39. By using Equations 30 to 39, we can get the value of $b_{0,0,0}$ easily by using excel spreadsheet.

$$
\begin{aligned}
b_{i, j, k}= & \frac{w_{i}-j}{w_{i}} b_{i-1,0, k}(1-d)[\alpha+(1-\alpha) \beta]=\frac{w_{i}-j}{w_{i}} b_{i, 0, k} \\
b_{0,0, k}= & (1-d)(1-\alpha)(1-\beta) P_{\text {coll }} \sum_{i=0}^{m} b_{i, 0, k-1} \\
& +(1-d)[\alpha+(1-\alpha) \beta] b_{m, 0, k-1} \\
= & (1-d)(1-\alpha)(1-\beta) P_{\text {coll }} \sum_{i=0}^{m} b_{i, 0, k-1}+Y b_{m, 0, k-1} \\
= & b_{0,0,0}\left[\left((1-d)(1-\alpha)(1-\beta) P_{\text {coll }} \sum_{i=0}^{m} Y^{i}\right)^{k}+\left(Y^{m+1}\right)^{k}\right] \\
= & b_{0,0,0}\left[\left((1-d)(1-\alpha)(1-\beta) P_{\text {coll }} \sum_{i=0}^{m} Y^{i}\right)^{k}+\left(Y^{m+1}\right)^{k}\right] \\
= & b_{0,0,0}\left[\left((1-d)(1-\alpha)(1-\beta) P_{\text {coll }} U\right)^{k}+Z^{k}\right]=b_{0,0,0}\left(X^{k}+Z^{k}\right)
\end{aligned}
$$


where $Y=(1-d)[a+(1-a) b] ; Z=Y^{m+1} ; \quad U=\left(\frac{1-Z}{1-Y}\right) ; \quad P_{\mathrm{pt}}=P$ (packet transmissions): and $X=(1-d)(1-a)(1-b) P_{\text {coll }} U$ for simplicity.

$$
\begin{aligned}
b_{i, 0, k} & =b_{i-1,0, k}(1-d)[\alpha+(1-\alpha) \beta]=((1-d)[\alpha+(1-\alpha) \beta])^{i} b_{0,0, k} \\
& =Y^{i} b_{0,0, k}=Y^{i} b_{0,0,0}\left(X^{k}+Z^{k}\right) \text { for } i \in(0, m), k \in(0, R)
\end{aligned}
$$

$P_{\text {randb }}=P($ perform random backoff $):$

$$
\begin{aligned}
& =\sum_{i=0}^{m} \sum_{j=0}^{w_{i}-1} \sum_{k=0}^{R} b_{i, j, k} \\
& =\sum_{i=0}^{m} \sum_{k=0}^{R} \frac{w_{i}}{2}((1-d)[\alpha+(1-\alpha) \beta])^{i} b_{0,0, k} \\
& =\sum_{i=0}^{m} \sum_{k=0}^{R} \frac{w_{i}}{2} Y^{i} b_{0,0, k} \\
& =\frac{w_{0}}{2} b_{0,0,0}\left(\frac{1-(X+Z)^{R}}{1-(X+Z)}-2 X Z\right)\left[\frac{1-(2 Y)^{R}}{1-(2 Y)}+4 Y^{3}+4 Y^{4}\right] \\
& =\frac{w_{0}}{2} b_{0,0,0} V Q
\end{aligned}
$$

where $V=\frac{1-(X+Z)^{R}}{1-(X+Z)}-2 X Z$ and $Q=\frac{1-(2 Y)^{R}}{1-(2 Y)}+4 Y^{3}+4 Y^{4}$ for simplicity.

$P_{\text {CCA1 }}=P$ (perform the first clear channel assessment):

$$
\begin{aligned}
& =\sum_{i=0}^{m} \sum_{k=0}^{R} b_{i,-1, k}=(1-d) \sum_{i=0}^{m} \sum_{k=0}^{R}((1-d)[\alpha+(1-\alpha) \beta])^{i} b_{0,0, k} \\
& =(1-d)(1-\alpha) \sum_{i=0}^{m} \sum_{k=0}^{R} Y^{i} b_{0,0, k} \\
& =b_{0,0,0}\left(\frac{1-(X+Z)^{R}}{1-(X+Z)}-2 X Z\right)\left(\frac{1-Z}{1-Y}\right)(1-d) \\
& =b_{0,0,0} V U(1-d)
\end{aligned}
$$

$P_{\mathrm{CCA} 2}=P($ perform the second clear channel assessment $):$

$$
\begin{aligned}
& =\sum_{i=0}^{m} \sum_{k=0}^{R} b_{i,-2, k}=(1-d)(1-\alpha) \sum_{i=0}^{m} \sum_{k=0}^{R}((1-d)[\alpha+(1-\alpha) \beta])^{i} b_{0,0, k} \\
& =(1-d)(1-\alpha) \sum_{i=0}^{m} \sum_{k=0}^{R} Y^{i} b_{0,0, k} \\
& =b_{0,0,0}\left(\frac{1-(X+Z)^{R}}{1-(X+Z)}-2 X Z\right)\left(\frac{1-Z}{1-Y}\right)(1-d)(1-\alpha) \\
& =b_{0,0,0} V U(1-d)(1-\alpha)
\end{aligned}
$$

$$
\begin{aligned}
& =\sum_{i=0}^{m} \sum_{k=0}^{R} b_{i,-3, k}=(1-d)(1-\alpha)(1-\beta) \sum_{i=0}^{m} \sum_{k=0}^{R} Y^{i} b_{0,0, k} \\
& =b_{0,0,0}\left(\frac{1-(X+Z)^{R}}{1-(X+Z)}-2 X Z\right)\left(\frac{1-Z}{1-Y}\right)(1-d)(1-\alpha)(1-\beta) \\
& =b_{0,0,0} V U(1-d)(1-\alpha)(1-\beta) \\
& P_{\text {suc }}=P(\operatorname{successful~packet~transmissions~}): \\
& =\sum_{i=0}^{m} \sum_{k=0}^{R} b_{S_{i, k}}=(1-d)(1-\alpha)(1-\beta)\left(1-P_{\text {coll }}\right) \sum_{i=0}^{m} \sum_{k=0}^{R} Y^{i} b_{0,0, k} \\
& =b_{0,0,0}\left(\frac{1-(X+Z)^{R}}{1-(X+Z)}-2 X Z\right)\left(\frac{1-Z}{1-Y}\right)(1-d)(1-\alpha)(1-\beta)\left(1-P_{\text {coll }}\right) \\
& =b_{0,0,0} V U(1-d)(1-\alpha)(1-\beta)\left(1-P_{\text {coll }}\right)
\end{aligned}
$$

$P_{\text {copt }}=P($ collided packet transmissions $):$

$$
\begin{aligned}
& =\sum_{i=0}^{m} \sum_{k=0}^{R} b_{C_{i, k}}=(1-d)(1-\alpha)(1-\beta) P_{\text {coll }} \sum_{i=0}^{m} \sum_{k=0}^{R} Y^{i} b_{0,0, k} \\
& =b_{0,0,0} X\left(\frac{1-(X+Z)^{R}}{1-(X+Z)}-2 X Z\right)=b_{0,0,0} X V
\end{aligned}
$$

$P_{\mathrm{dtx}}=P($ deferred transmission $):$

$$
\begin{aligned}
& =\sum_{i=0}^{m} \sum_{k=0}^{R} b_{D_{i, k}}=d \sum_{i=0}^{m} \sum_{k=0}^{R}((1-d) \alpha+(1-d)(1-\alpha) \beta)^{i} b_{0,0, k} \\
& =d \sum_{i=0}^{m} \sum_{k=0}^{R}((1-d)(\alpha+(1-\alpha) \beta))^{i} b_{0,0, k}=d \sum_{i=0}^{m} \sum_{k=0}^{R} Y^{i} b_{0,0, k} \\
& =b_{0,0,0} d\left(\frac{1-(X+Z)^{R}}{1-(X+Z)}-2 X Z\right)\left(\frac{1-Z}{1-Y}\right)=b_{0,0,0} d V U
\end{aligned}
$$




$$
\begin{aligned}
& =\sum_{i=0}^{m} \sum_{k=0}^{R} b_{\mathrm{SG}_{i, k}}=d P_{\mathrm{GTS}} \sum_{i=0}^{m} \sum_{k=0}^{R}((1-d)(\alpha+(1-\alpha) \beta))^{i} b_{0,0, k} \\
& =d P_{\mathrm{GTS}} \sum_{i=0}^{m} \sum_{k=0}^{R} Y^{i} b_{0,0, k} \\
& =b_{0,0,0} d P_{\mathrm{GTS}}\left(\frac{1-(X+Z)^{R}}{1-(X+Z)}-2 X Z\right)\left(\frac{1-Z}{1-Y}\right) \\
& =b_{0,0,0} d P_{\mathrm{GTS}} V U
\end{aligned}
$$

$$
\begin{aligned}
& P_{\mathrm{idle}}=(1-q) \sum_{i=0}^{m} \sum_{k=0}^{R} b_{S_{i, k}}+(1-q) \sum_{i=0}^{m} \sum_{k=0}^{R} b_{\mathrm{SG}_{i, k}}+(1-q) \sum_{i=0}^{m} b_{C_{i, R}}+d(1-q)\left(1-P_{\mathrm{GTS}}\right) \sum_{i=0}^{m} b_{i, 0,0} \\
& +(1-q)((1-d)[\alpha+(1-\alpha) \beta]) b_{m, 0, R}+(1-q) P_{\text {idle }} \\
& P_{\text {idle }}=\frac{(1-q)}{q}\left[\sum_{i=0}^{m} \sum_{k=0}^{R} b_{S_{i, k}}+\sum_{i=0}^{m} \sum_{k=0}^{R} b_{\mathrm{SG}_{i, k}}+\sum_{i=0}^{m} b_{C_{i, R}}+d\left(1-P_{\mathrm{GTS}}\right) \sum_{i=0}^{m} b_{i, 0,0}+Y b_{m, 0, R}\right] \\
& =\frac{(1-q)}{q} b_{0,0,0}\left[\begin{array}{l}
\left(\frac{1-(X+Z)^{R}}{1-(X+Z)}-2 X Z\right)\left(\frac{1-(Y)^{m+1}}{1-(Y)}\right)(1-d)(1-\alpha)(1-\beta)\left(1-P_{\text {coll }}\right)+ \\
\left(\frac{1-(X+Z)^{R}}{1-(X+Z)}-2 X Z\right)\left(\frac{1-(Y)^{m+1}}{1-(Y)}\right) d P_{\mathrm{GTS}}+ \\
\left(X^{2}+Z^{2}\right)(1-d)(1-\alpha)(1-\beta) P_{\text {coll }}\left(\frac{1-Y^{m+1}}{1-Y}\right)+ \\
d\left(1-P_{\mathrm{GTS}}\right)\left(\frac{1-Y^{m+1}}{1-Y}\right)+Y^{m+1}\left(X^{2}+Z^{2}\right)
\end{array}\right] \\
& =\frac{(1-q)}{q} b_{0,0,0}\left[\begin{array}{c}
V U(1-d)(1-\alpha)(1-\beta)\left(1-P_{\mathrm{coll}}\right)+V U d P_{\mathrm{GTS}} \\
+\left(X^{2}+Z^{2}\right) X+d\left(1-P_{\mathrm{GTS}}\right) U+Z\left(X^{2}+Z^{2}\right)
\end{array}\right] \\
& =\frac{(1-q)}{q} b_{0,0,0}\left[\begin{array}{l}
V U\left[(1-d)(1-\alpha)(1-\beta)\left(1-P_{\text {coll }}\right)+d P_{\mathrm{GTS}}\right] \\
+\left(X^{2}+Z^{2}\right)(X+Z)+d\left(1-P_{\mathrm{GTS}}\right) U
\end{array}\right]
\end{aligned}
$$

$$
\begin{aligned}
\sum_{i=0}^{m} \sum_{j=0}^{w_{i-1}} \sum_{k=0}^{R} b_{i, j, k} & +\sum_{i=0}^{m} \sum_{k=0}^{R} b_{i,-1, k}+\sum_{i=0}^{m} \sum_{k=0}^{R} b_{i,-2, k} \\
& +\sum_{i=0}^{m} \sum_{k=0}^{R} b_{i,-3, k}+\sum_{i=0}^{m} \sum_{k=0}^{R} b_{S_{i, k}} \\
& +\sum_{i=0}^{m} \sum_{k=0}^{R} b_{C_{i, k}}+\sum_{i=0}^{m} \sum_{k=0}^{R} b_{D_{i, k}} \\
& +\sum_{i=0}^{m} \sum_{k=0}^{R} b_{\mathrm{SG}_{i, k}}+P_{\mathrm{idle}}=1
\end{aligned}
$$

Let $\phi_{1}$ and $\phi_{2}$ be the conditional probabilities that a tagged node will be at one of the $\mathrm{CCA}_{1}$ states after backoff and at one of the $\mathrm{CCA}_{2}$ states after sensing channel idle in the $\mathrm{CCA}_{1}$, which can be obtained by Equations 40 and 41, respectively. Let us denote $\tau$ be the probability that a device node can transmit a packet, i.e., the device node is in one of the $\mathrm{CCA}_{1}$ states and senses the $\mathrm{CCA}_{2}$ is idle, while the other nodes are not in the $\mathrm{CCA}_{1}$ state, which can be calculated by Equation 42 . Let us denote $N_{\text {GTS }}$ and $N_{\text {WGTS }}$ as the number of nodes to be and not to be allocated GTS slots, respectively.

Since $\alpha$ is the probability of the $\mathrm{CCA}_{1}$ busy, we can also consider with the transmission of the data packets and GTS request packets. Let $\alpha_{\text {data }}$ and $\alpha_{\text {request }}$ be the probabilities that $C \mathrm{~A}_{1}$ is busy for sending data packets and GTS request packets, which can be obtained by Equations 43 and 44 , respectively. Similarly, let $\beta_{\text {data }}$ and $\beta_{\text {request }}$ be the probabilities that $\mathrm{CCA}_{2}$ is busy for sending data packets and GTS request packets, which can be obtained by Equations 45 and 46, respectively. Therefore, $\alpha$ and $\beta$ can be obtained by Equations 47 and 48, respectively. Finally, the previous mentioned probabilities of $P_{\text {coll }}, P_{\text {fail } 1}$, and $P_{\text {fail } 2}$ can be expressed by Equations 49 to 51, respectively. 


$$
\begin{aligned}
\phi_{1} & =\frac{\sum_{i=0}^{m} \sum_{k=0}^{R} b_{i,-1, k}}{\sum_{i=0}^{m} \sum_{k=0}^{R} b_{i,-1, k}+\sum_{i=0}^{m} \sum_{j=0}^{w_{i}-1} \sum_{k=0}^{R} b_{i, j, k}} \\
& =\frac{\left(\frac{1-(X+Z)^{R+1}}{1-(X+Z)}-2 X Z\right)\left(\frac{1-Z}{1-Y}\right)(1-d)}{\left(\frac{1-(X+Z)^{R+1}}{1-(X+Z)}-2 X Z\right)\left[\left(\frac{1-Z}{1-Y}\right)(1-d)+\frac{w_{0}}{2}\left(\frac{1-(2 Y)^{R+1}}{1-(2 Y)}+4 Y^{3}+4 Y^{4}\right)\right]} \\
& =\frac{V^{\prime} U(1-d)}{V^{\prime}\left[U(1-d)+\frac{w_{0}}{2} Q^{\prime}\right]}
\end{aligned}
$$

where $V^{\prime}=\frac{1-(X+Z)^{R+1}}{1-(X+Z)}-2 X Z$ and $Q^{\prime}=\frac{1-(2 Y)^{R+1}}{1-2 Y}+4 Y^{3}+4$

$Y^{4}$ for simplicity.

$$
\begin{aligned}
& \phi_{2}=\frac{\sum_{i=0}^{m} \sum_{k=0}^{R} b_{i,-2, k}}{\sum_{i=0}^{m} \sum_{k=0}^{R} b_{i,-1, k}+\sum_{i=0}^{m} \sum_{k=0}^{R} b_{i,-2, k}+\sum_{i=0}^{m} \sum_{j=0}^{w_{i}-1} \sum_{k=0}^{R} b_{i, j, k}} \\
& =\frac{\left(\frac{1-(X+Z)^{R+1}}{1-(X+Z)}-2 X Z\right)\left(\frac{1-Z}{1-Y}\right)(1-d)(1-\alpha)}{\left(\frac{1-(X+Z)^{R+1}}{1-(X+Z)}-2 X Z\right)\left[\left(\frac{1-Z}{1-Y}\right)(1-d)(2-\alpha)+\left(\frac{w_{0}}{2}\left(\frac{1-(2 Y)^{R+1}}{1-2 Y}+4 Y^{3}+4 Y^{4}\right)\right)\right]} \\
& =\frac{V^{\prime} U(1-d)(1-\alpha)}{V^{\prime}\left[U(1-d)(2-\alpha)+\frac{w_{0}}{2} Q^{\prime}\right]} \\
& \tau=\phi_{1}\left(1-\phi_{1}\right)^{N_{\mathrm{WGTS}}-1}(1-\alpha)(1-\beta) \\
& \alpha_{\text {data }}=(1-P) \phi_{1}\left(1-(1-\tau)^{\left(N_{\mathrm{WGTS}}-1\right)}\right) \\
& \alpha_{\text {request }}=P \phi_{1}\left(1-(1-\tau)^{\left(N_{\mathrm{WGTS}}-1\right)}\right) \\
& \beta_{\mathrm{data}}=(1-P) \phi_{2}\left(1-(1-\tau)^{\left(N_{\mathrm{WGTS}}-1\right)}\right) \\
& P_{\text {coll }}=N_{\mathrm{WGTS}} \tau\left(1-(1-\tau)^{\left(N_{\mathrm{WGTS}}-1\right)}\right) \\
& P_{\text {fail }}=\sum_{i=0}^{m} b_{C_{i, R}}=b_{0,0,0}\left(X^{R}+Z^{R}\right) X \\
& P_{\text {fail2 }}=b_{m, 0, R}(1-d)(\alpha+(1-\alpha) \beta) \\
& =b_{0,0,0} Y^{m}\left(X^{R}+Z^{R}\right)(1-d)(\alpha+(1-\alpha) \beta)
\end{aligned}
$$$$
\beta_{\text {request }}=P \phi_{2}\left(1-(1-\tau)^{\left(N_{\mathrm{WGTS}}-1\right)}\right)
$$$$
\alpha=\alpha_{\text {data }}+\alpha_{\text {request }}=\phi_{1}\left(1-(1-\tau)^{\left(N_{\mathrm{WGTS}}-1\right)}\right)
$$$$
\beta=\beta_{\text {data }}+\beta_{\text {request }}=\phi_{2}\left(1-(1-\tau)^{\left(N_{\mathrm{WGTS}}-1\right)}\right)
$$

Let denote $P_{\text {cr }}$ to be the probability of collision transmission after $j$ attempts (probability of packet being dropped due to collision retransmission), which can be calculated by Equation 52 . Let $P_{\text {dropWGTS }}$ and $P_{\text {sucWGTS }}$ be the probabilities of non-GTS packets being dropped and successful transmission from device node to its coordinator, which can be obtained by 
Equations 53 and 54, respectively. Let us also denote $N_{\text {WGTSrecvcoord }}$ and $T_{\text {sim }}$ to be the number of nonGTS packets received by the network coordinator and time of simulation, which can be calculated by Equation 55. Therefore, the goodput of SUDAS CAP in the network, denoted by $S_{\text {CAPsudaslength }}$, can be calculated by Equation 56 .

$$
\begin{aligned}
& P_{\text {cr }}=\sum_{k=1}^{R}\left(P_{\text {coll }}\right)^{k} \\
& P_{\text {dropWGTS }}=P_{\text {cr }}+P_{\text {fail } 1}+P_{\text {fail } 2} \\
& \mathrm{P}_{\text {sucWGTS }}=1-\mathrm{P}_{\text {dropWGTS }} \\
& N_{\text {WGTSreccoord }}=\frac{(1-P) \times \lambda_{n} \times N_{\text {WGTS }} \times\left(1-P_{\text {dropWGTS }}\right) \times T_{\text {sim }}}{L_{\text {data }}} \\
& S_{\text {CAPsudaslength }}=\frac{N_{\text {WGTSrecvcoord }} \times L_{\text {data }}}{N_{\text {beacon }} \times B I_{\text {coord }}}
\end{aligned}
$$

Let us denote $R_{\text {req }}$ and $N_{\text {req }}$ to be the GTS request rate of device node and the number of packets per request, respectively, then $P_{\mathrm{GTS}}$ can be calculated by Equation 57. Let $P_{\text {tsg }}$ be the probability of the successful GTS transmission, which can be obtained by Equation 58, where $P_{\mathrm{sg}}$ has been done by Equation 37 . Let also denote $N_{\text {GTSrecvcoord }}$ and $S_{\text {CFPsudaslength }}$ be the number of GTS packets received by the network coordinator and the goodput of SUDAS CFP in the network, which can be calculated by Equations 59 and 60, respectively. Finally, the total goodput in the network, denoted by $S_{\text {total }}$, can be calculated by Equation 61 .

$$
\begin{aligned}
& P_{\mathrm{GTS}}=R_{\text {req }} \times N_{\text {req }} \times\left(\frac{T x_{n}}{T_{\mathrm{sd}}}\right) \\
& P_{\mathrm{tsg}}=P_{\mathrm{sg}} \times\left(\frac{P_{\mathrm{GTS}}+1}{P_{\mathrm{sg}}}\right) \times(1-d) \\
& N_{\mathrm{GTSrecvcoord}}=\frac{P \times \lambda_{n} \times N_{\mathrm{GTS}} \times P_{\mathrm{tsg}} \times T_{\mathrm{sim}}}{L_{\mathrm{data}}}
\end{aligned}
$$

$$
S_{\text {CFPsudaslength }}=\frac{N_{\text {GTSrecccoord }} \times L_{\text {data }}}{N_{\text {beacon }} \times B I_{\text {coord }}}
$$

$$
S_{\text {total }}=\frac{\left(N_{\text {WGTSrecvcoord }}+N_{\text {GTSrecvcoord }}\right) \times L_{\text {data }}}{N_{\text {beacon }} \times B I_{\text {coord }}}
$$

Let $N_{\text {beacon }}$ and $T_{\mathrm{q}}$ be the number of beacons and the time to transmit one data packet and receive ACK packet in $\mathrm{CAP}_{\text {sudaslength}}$, which can be obtained by Equations 62 and 63, respectively. Let us also denote bandwidth utilization $(\mathrm{BU})_{\mathrm{CAPsudaslength}} \mathrm{BU}_{\mathrm{CFPsudaslength}}$ and $\mathrm{BU}_{\text {sudastotal }}$ to be the $\mathrm{BUs}$ for CAP, CFP, and total average amount in the network, which can be obtained by Equations 64 to 66, respectively.

$$
\begin{aligned}
& N_{\text {beacon }}=\frac{T_{\text {sim }}}{T_{\text {sd }}} \\
& T_{q}=2 T_{t x \mathrm{CCA}}+T_{f} \\
& \mathrm{BU}_{\text {CAPsudaslength }}=\frac{N_{\text {WGTSrecvcoord }} \times T_{q}}{N_{\text {beacon }} \times \mathrm{CAP}_{\text {sudaslength }}} \\
& \mathrm{BU}_{\text {CFPsudaslength }}=\frac{N_{\text {GTSrecvcoord }} \times T_{f}}{\left(T_{\text {sudas }} \sum_{n=1}^{N_{\text {GTS }}} N \text { sudas }_{n}\right) \times N_{\text {beacon }}} \\
& \mathrm{BU}_{\text {sudastotal }}=\frac{\mathrm{BU}_{\mathrm{CAP} \text { sudaslength }}+\mathrm{BU}_{\mathrm{CFPsudaslength}}}{2}
\end{aligned}
$$

Let $T_{\text {eCAPsudaslength }}$ and $L_{\text {delay }}$ be the estimated remaining $\mathrm{CAP}_{\text {sudaslength }}$ in time (seconds) and length (bits), which can be calculated by Equations 67 to 68, respectively. Let us denote $E_{\mathrm{dev}} E_{\mathrm{coord}}$ and $E_{\text {total }}$ to be the energy consumptions by device nodes, coordinator node, and total amount in the star topology, which can be calculated by Equations 69 to 71 , respectively, where $P W R_{\text {idle, }} P W R_{t x}$, and $\mathrm{PWR}_{\mathrm{rx}}$ are the power consumptions for idle, transmitting a packet and receiving a packet, respectively; and $L_{\mathrm{CCA}}, L_{\text {beacon }}$, and $L_{\text {request }}$ are the transmission lengths (bits) of CCA, beacon, and GTS request (72 bits), respectively. Let us denote $D_{\text {node }}$ to be the distance between a device node and its coordinator.

$$
\begin{aligned}
T_{\text {eCAPsudaslength }}= & P_{\mathrm{dtx}} \times \mathrm{CAP}_{\text {sudaslength }}=b_{0,0,0} \times V \times U \\
& \times d \times \mathrm{CAP}_{\text {sudaslength }}
\end{aligned}
$$

$$
L_{\text {delay }}=T_{\text {eCAPsudaslength }} \times R_{b}
$$




$$
\begin{aligned}
& E_{\mathrm{dev}}=\left(\mathrm{PWR}_{\mathrm{idle}} \sum_{i=0}^{m} \sum_{j=0}^{w_{i}-1} \sum_{k=0}^{R} b_{i, j, k} \mathrm{SD}_{\mathrm{Coord}} \frac{T_{\text {sim }}}{\mathrm{BI}_{\text {Coord }}}\left(N_{\mathrm{WGTS}}\right)\right)+ \\
& \left(2 \operatorname{PWR}_{t x} \frac{(1-P)\left(\lambda_{n}\right)}{\operatorname{CAP}_{\text {sudaslength }}} \frac{L_{\mathrm{CCA}}}{R_{b}} \sum_{i=0}^{m} \sum_{k=0}^{R}\left(b_{i,-1, k}+b_{i,-2, k}\right) T_{\text {sim }} D_{\text {node }}\left(N_{\text {WGTS }}\right)\right)+ \\
& \left(\operatorname{PWR}_{t x} \frac{(1-P)\left(\lambda_{n}\right)}{\mathrm{CAP}_{\text {sudaslength }}} \frac{L_{\text {data }}}{R_{b}} P_{\text {sucWGTS }} T_{\text {sim }} D_{\text {node }}\left(N_{\text {WGTS }}\right)\right)+\left(\operatorname{PWR}_{t x} \frac{(P)\left(\lambda_{n}\right)}{\operatorname{CAP}_{\text {sudaslength }}} \frac{L_{\text {request }}}{R_{b}} P_{\text {GTS }} T_{\text {sim }} D_{\text {node }}\left(N_{\text {GTS }}\right)\right)+ \\
& \left(\operatorname{PWR}_{t x} \frac{(P)\left(\lambda_{n}\right)}{\mathrm{CFP}_{\text {sudaslength }}} \frac{L_{\text {data }}}{R_{b}} P_{\mathrm{GTS}} T_{\text {sim }} D_{\text {node }}\left(N_{\mathrm{GTS}}\right)\right)+\left(\operatorname{PWR}_{t x} \frac{(1-P)\left(\lambda_{n}\right)}{\mathrm{CAP}_{\text {sudaslength }}} \frac{L_{\text {delay }}}{R_{b}} P_{\mathrm{dtx}} T_{\text {sim }}\left(N_{\mathrm{WGTS}}\right)\right)+ \\
& \left(\mathrm{PWR}_{\text {idle }} P_{\text {idle }} S D_{\text {Coord }} \frac{T_{\text {sim }}}{\mathrm{BI}_{\text {Coord }}}\left(N_{\text {total }}\right)\right)+\left(\mathrm{PWR}_{r x} \frac{L_{\text {beacon }}}{R_{b}} \frac{T_{\text {sim }}}{\mathrm{BI}_{\text {coord }}}\left(N_{\text {total }}\right)\right)+ \\
& \left(\operatorname{PWR}_{r x} \frac{(1-P)\left(\lambda_{n}\right)}{\mathrm{CAP}_{\text {sudaslength }}} \frac{L_{\text {ack }}}{R_{b}} P_{\text {sucWGTS }} T_{\text {sim }}\left(N_{\text {WGTS }}\right)\right)+\left(\operatorname{PWR}_{r x} \frac{(P)\left(\lambda_{n}\right)}{\mathrm{CFP}_{\text {sudaslength }}} \frac{L_{\text {ack }}}{R_{b}} P_{\text {GTS }} T_{\text {sim }}\left(N_{\text {GTS }}\right)\right)
\end{aligned}
$$

$$
\begin{aligned}
& E_{\text {Coord }}=\left(\mathrm{PWR}_{\text {idle }} P_{\text {idle }} \mathrm{SD}_{\text {Coord }} \frac{T_{\text {sim }}}{\mathrm{BI}_{\text {Coord }}}\right) \\
& +\left(\mathrm{PWR}_{t x} \frac{L_{\text {beacon }}}{R_{b}} \frac{T_{\text {sim }}}{\mathrm{BI}_{\text {Coord }}} D_{\text {node }}\right) \\
& +\left(\text { PWR }_{\mathrm{rx}} \frac{(1-P)\left(\lambda_{n}\right)}{\mathrm{CAP}_{\text {sudaslength }}} \frac{L_{\mathrm{data}}}{R_{b}} P_{\text {sucWGTS }} T_{\text {sim }} N_{\text {WGTS }}\right) \\
& +\left(\mathrm{PWR}_{r x} \frac{(P)\left(\lambda_{n}\right)}{\mathrm{CAP}_{\text {sudaslength }}} \frac{L_{\text {request }}}{R_{b}} P_{\mathrm{GTS}} T_{\text {sim }} N_{\mathrm{GTS}}\right) \\
& +\left(\mathrm{PWR}_{r x} \frac{(P)\left(\lambda_{n}\right)}{\mathrm{CFP}_{\text {sudaslength }}} \frac{L_{\mathrm{data}}}{R_{b}} P_{\mathrm{GTS}} T_{\text {sim }} N_{\mathrm{GTS}}\right) \\
& +\left(\mathrm{PWR}_{t x} \frac{(1-P)\left(\lambda_{n}\right)}{\mathrm{CAP}_{\text {sudaslength }}} \frac{L_{\text {ack }}}{R_{b}} P_{\text {sucWGTS }} T_{\text {sim }} D_{\text {node }} N_{\text {WGTS }}\right) \\
& +\left(\operatorname{PWR}_{t x} \frac{(P)\left(\lambda_{n}\right)}{\mathrm{CFP}_{\text {sudaslength }}} \frac{L_{\text {ack }}}{R_{b}} P_{\text {GTS }} T_{\text {sim }} D_{\text {node }} N_{\text {GTS }}\right)
\end{aligned}
$$

$$
E_{\mathrm{total}}=E_{\mathrm{dev}}+E_{\mathrm{coord}}
$$

The energy consumption of device node consists of ten parts as shown in Equation 69. The first part is energy consumption for backoff. The second part is energy consumption for CCA transmission. The third part is energy consumption for data packet transmission using CSMA/CA in CAP. The fourth part is energy consumption for request GTS. The fifth part is energy consumption for data packet transmission using GTS in CFP. The sixth part is energy consumption for waiting due to deferred transmission. The seventh part is energy consumption for idle. The eighth part is energy consumption for receiving beacon. The ninth part is energy consumption for receiving ACK if transmitting packet is successful, and the tenth part is energy consumption for ACK if transmission packet using GTS is successful.

The energy consumption of coordinator node consists of seven parts as shown in Equation 70. The first part is energy consumption for idle. The second part is energy consumption for transmission beacon. The third part is energy consumption for receiving data at CSMA/CA in CAP. The fourth part is energy consumption for receiving request for GTS allocation. The fifth part is energy consumption for receiving data at GTS in CFP. The sixth part is energy consumption for transmitting ACK, if receiving packet successful in CAP. The seventh part is energy consumption for transmitting ACK, if receiving packet GTS is successful in CFP. Finally, the total energy consumption in networks consist of the energy consumption by devices node and coordinator node as shown in Equation 71.

\section{Simulation and analysis results}

In this section, simulation experiments for SUDAS are performed by using the extended Castalia simulator to validate the analysis and performance evaluation. The performance of SUDAS is compared with NGAS, ANBU, and the IEEE 802.15.4 standard, which include the analytical (ana) and simulation (sim) results. We consider a star topology with one PAN coordinator and 20 device nodes, where $D_{\text {node }}$ is equal to $10 \mathrm{~m}$. To simulate the performance of power consumption, we consider the radio parameters of Chipcon's CC2420 2.4 $\mathrm{GHz}$ for the IEEE 802.15.4 RF transceiver [32], where the transmitting power $\mathrm{PWR}_{\mathrm{tx}}$, the receiving power $\mathrm{PWR}_{\mathrm{rx}}$, and the idle power $\mathrm{PWR}_{\text {idle }}$ are $31.32 \mathrm{~mW}, 35.28$ $\mathrm{mW}$, and $712 \mu \mathrm{W}$, respectively [33]. The BO and SO settings follow the IEEE 802.15.4 standard and the proposed SUDAS algorithm, which are fixed to be six. We compute the probability of successful packet transmission in CAP, network goodput, average bandwidth utilization (BU), and total network energy consumption, where traffic load varies from 0.1 to 1 (full loaded). Table 1 summarizes the simulation parameters.

Figure 6 shows the probability of successful transmission arriving at the PAN coordinator against the traffic load by analytical and simulation. In CFP, nodes do not 
Table 1 The simulation parameters

\begin{tabular}{|c|c|}
\hline Parameter & Value \\
\hline Physical data rate & $250 \mathrm{kbps}$ \\
\hline Packet length ( $\left.L_{\text {data }}\right)$ & 560 bits \\
\hline UBP & 80 bits \\
\hline NumSuperframeSlots & 16 \\
\hline MacPacketOverhead & 112 bits \\
\hline ACK length $\left(L_{a c k}\right)$ & 88 bits \\
\hline$D_{\text {node }}$ & $10 \mathrm{~m}$ \\
\hline$P W R_{\mathrm{tx}}$ & $31.32 \mathrm{~mW}$ \\
\hline$P W R_{r x}$ & $35.28 \mathrm{~mW}$ \\
\hline$P W R_{\text {idle }}$ & $712 \mu \mathrm{W}$ \\
\hline $\mathrm{BO}=\mathrm{SO}$ & 6 \\
\hline $\mathrm{BE}_{\min }$ & 3 \\
\hline $\mathrm{BE}_{\max }$ & 5 \\
\hline
\end{tabular}

compete with each other and each node has the dedicated slots, hence we only consider part of CAP in this article. The proposed SUDAS algorithm has higher probability of successful transmissions than those of NGAS, ANBU, and IEEE 802.15.4 standard because the length of $\mathrm{CAP}_{\text {sudaslength }}$ is longer than those of $\mathrm{CAP}_{\mathrm{NGAS}}$, $\mathrm{CAP}_{\mathrm{ANBU}}$, and $\mathrm{CAP}_{\text {standard }}$, respectively.

Figure 7 shows the network goodput against traffic load. The network goodput obtained by simulation is very close to that obtained by the analytical model. It is obvious the network goodput of SUDAS is higher than those of the other algorithms. In the light traffic load (i.e., traffic load is equal to 0.1 and 0.2 ), the network goodput of SUDAS is almost the same as those of NGAS, ANBU, and IEEE standard; however, SUDAS outperforms the other algorithms as the traffic load increases. The average goodput of SUDAS increases by $8.30 \%, 14.23 \%$, and $19.21 \%$ compared to NGAS, ANBU, and IEEE 802.15.4 standard, respectively.

Figure 8 shows the average BU against traffic load. The average bandwidth utilization of SUDAS has better efficiency than those of other algorithms. The average bandwidth utilization of SUDAS increases by $20.41 \%$, $33.80 \%$, and $41.65 \%$ compared to NGAS, ANBU, and IEEE 802.15.4 standard, respectively. SUDAS can improve the average bandwidth utilization because the size of slot is adjustable with the data packet to be transmitted, i.e., SUDAS can reduce the waste of bandwidth.

Figure 9 shows the network energy consumption against traffic load. The average energy consumption of SUDAS reduces by $5.65 \%, 12.94 \%$, and $26.15 \%$ compared to NGAS, ANBU, and IEEE standard, respectively. SUDAS consumes lesser network energy than those of other algorithms, because $\mathrm{CAP}_{\text {sudaslength }}$ is longer than those of other algorithms. Moreover, SUDAS has greater probability of successful transmission than those of other algorithms, especially in heavy traffic load, which means that SUDAS minimizes the energy consumption when retransmitting data packet. The energy consumption is obtained by summing the energy consumption of PAN coordinator and all of device nodes in the network.

\section{Conclusions}

In this article, SUDAS is proposed to improve the IEEE 802.15.4 medium access control, which analyzes not only

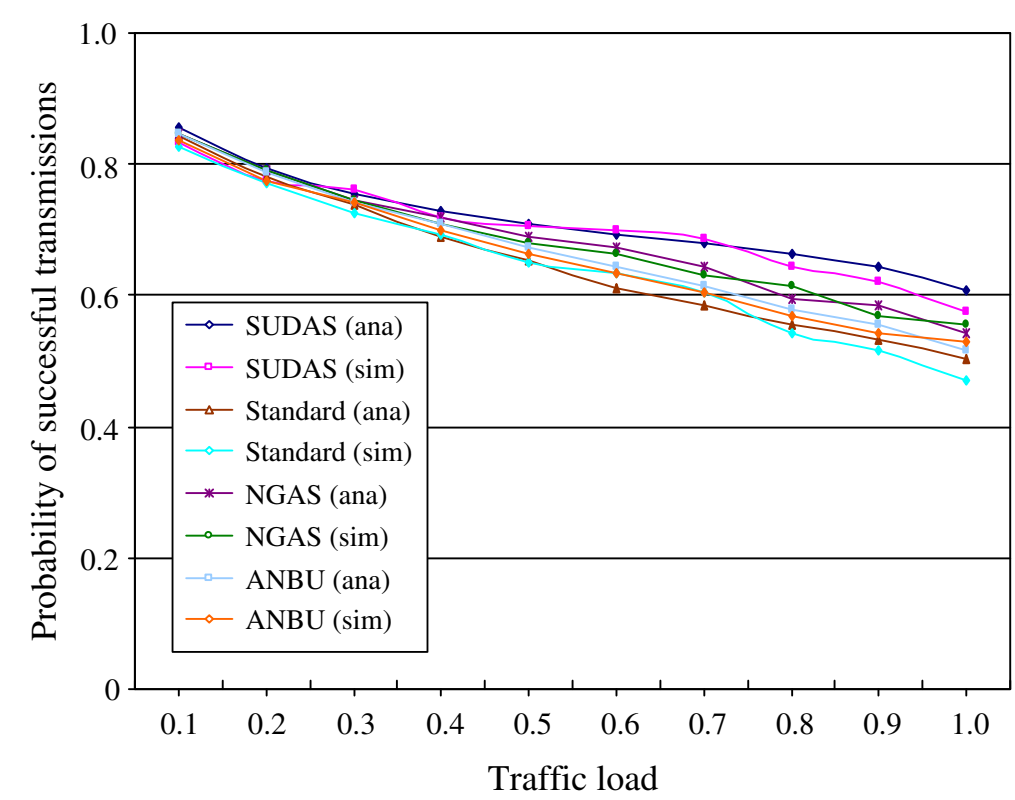

Figure 6 The probability of successful transmission against traffic load. 


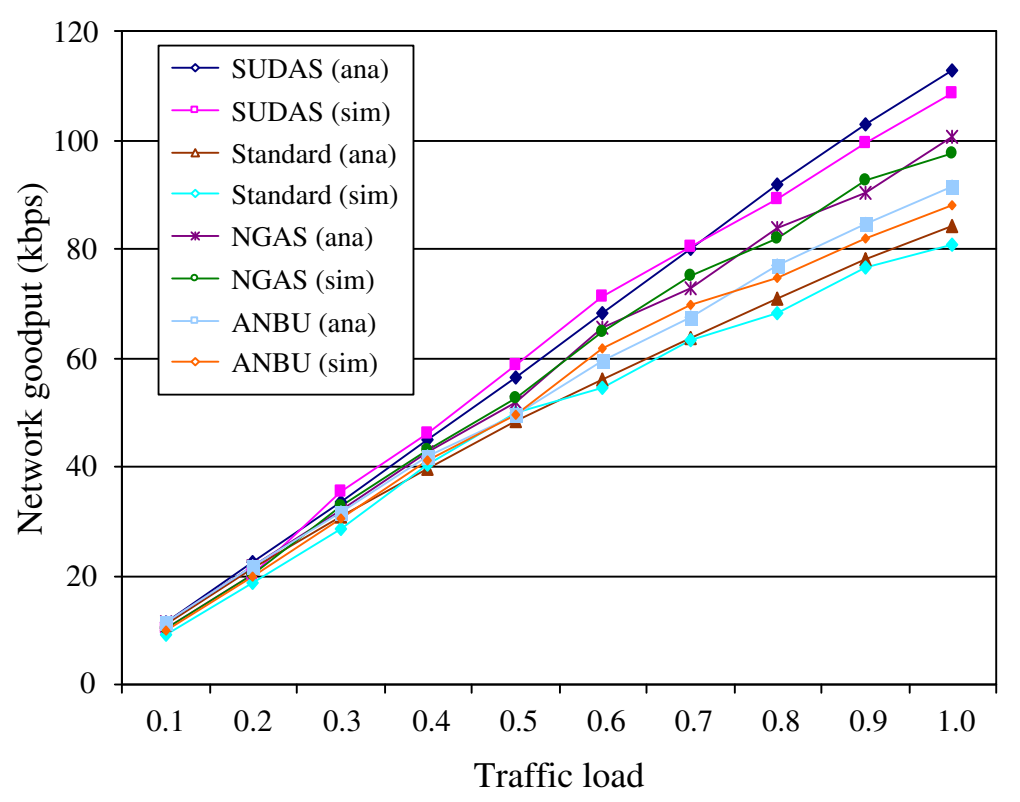

Figure 7 The network goodput against traffic load.

CAP but also CFP. SUDAS performs with the adjustable length of the slot in the superframe duration based on the length of data packet, so that it can accurately decide for the starting time, and the GTS length to be allocated for the requested devices to alleviate the waste of GTS bandwidth utilization. SUDAS is expected to effectively allocate GTS to the requested devices, because the length of $\mathrm{CAP}_{\text {sudaslength }}$ is longer than those of other algorithms.
This paper also presented a comprehensive Markov chain analysis of IEEE 802.15.4, specifically for star topology, to predict the probability of successful transmission, the network goodput, average bandwidth utilization, as well as the network energy consumption. The validity of the analytical model is shown by closely matching its predictions of the simulation results. The analytical model and simulation experiment results show that the performance of SUDAS is better than those of other

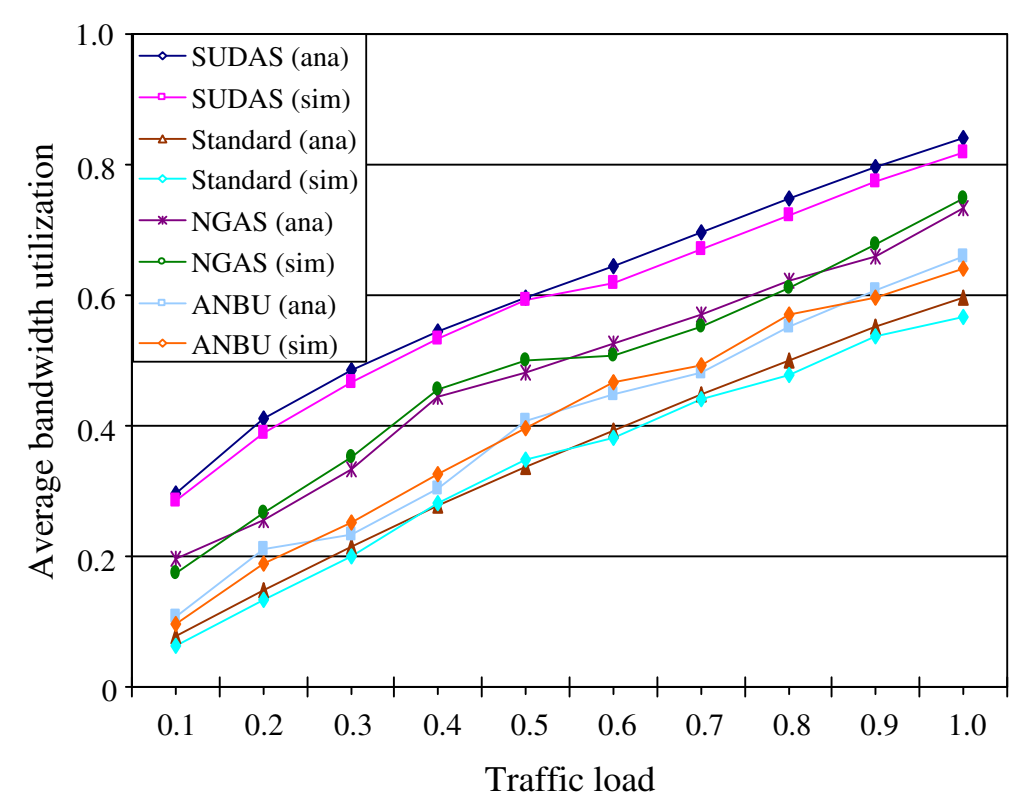

Figure 8 The average bandwidth utilization against traffic load. 


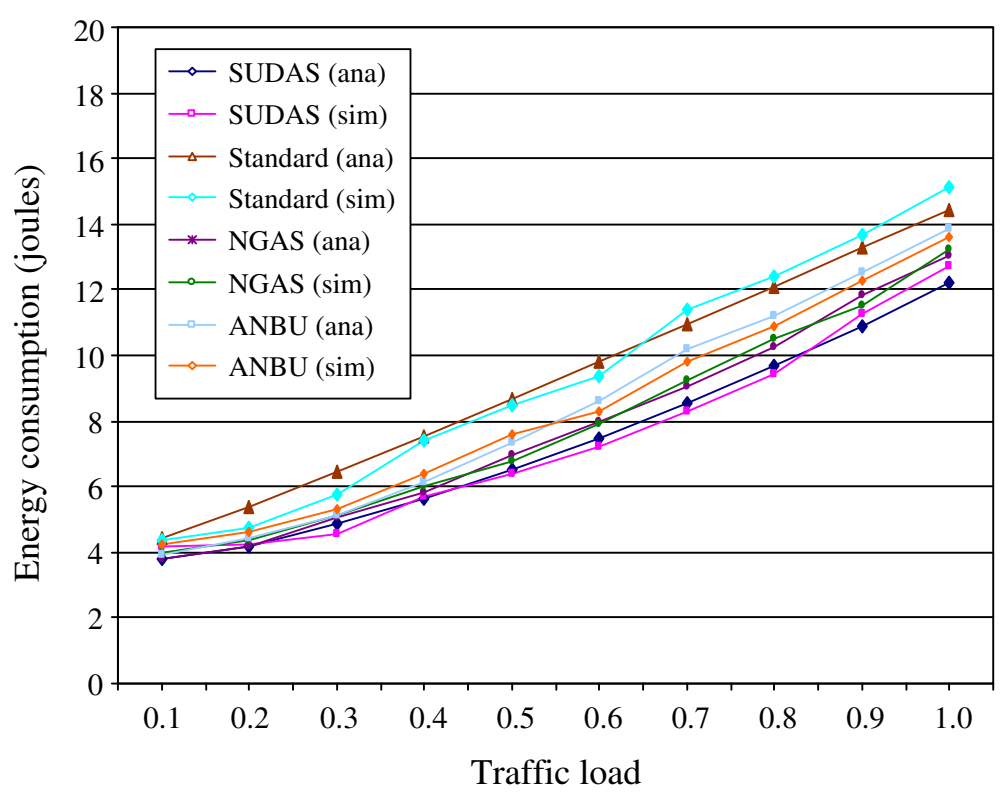

Figure 9 The total of network energy consumption against traffic load.

algorithms in terms of the probability of successful transmission, network goodput, average bandwidth utilization, and energy consumption.

\subsection{Summary of parameters}

aBaseSlotDuration, the minimum Number of symbols in a slot; aBaseSuperframeDuration, the minimum Number of symbols in an active period; aMinCAPlength, the minimum value of CAP length; aNumSuperframeSlots, number of superframe slots; macMaxBE, the maximum value of backoff exponent; macMaxCSMABackoff, the

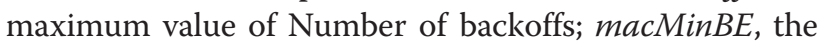
minimum value of backoff exponent.

\subsection{Summary of symbols}

adjslot, the integer value that will be used as the adjustment become new smaller adjustment time of one slot duration; $b_{\mathrm{Ci}, \mathrm{k}}$, stationary probability of the collision at the states of $C_{\mathrm{i}, \mathrm{k}} ; b_{\mathrm{Di}, \mathrm{k}}$, stationary probability of waiting due to deferred transmission at state of $D_{\mathrm{i}, \mathrm{k}} ; b_{i,-1, \mathrm{k}}$, stationary probability for $\mathrm{CCA}_{1}$ at the stochastic state $(s(t)=i$ and $r(t)=k) ; b_{i,-2, k}$, stationary probability for $\mathrm{CCA}_{2}$ at the stochastic state $(s(t)=i$ and $r(t)=k) ; b_{i,-3, k}$, stationary probability for packet transmission at the stochastic state $(s(t)=i$ and $r(t)=k) ; b_{i, j, k}$, stationary probability at the stochastic state $(\mathrm{s}(\mathrm{t})=\mathrm{i}, \mathrm{c}(\mathrm{t})=\mathrm{j}$ and $\mathrm{r}(\mathrm{t})=\mathrm{k})$; $\mathrm{BI}_{\text {coord, }}$, beacon interval of coordinator; $\mathrm{BO}_{\text {coord }}$, beacon order for coordinator; $b_{\mathrm{SG} i, k}$, stationary probability of successful request GTS allocation at state of $\mathrm{SG}_{i, k} ; b_{S i, k}$, stationary probability of the successful transmission at the states of $S_{i, k} ; \mathrm{BU}_{\mathrm{CAPsudaslength}}$, the bandwidth utilization for CAPsudaslength; $\mathrm{BU}_{\mathrm{CFPsudaslength}}$, the bandwidth utilization for $\mathrm{CFP}_{\text {sudaslength }}$ B $\mathrm{BU}_{\text {sudastotal, }}$, the total average bandwidth utilization in the network; $c(t)$, backoff counter in stochastic state; $\mathrm{CAP}_{\mathrm{ANBU}}$, the time of CAP period in ANBU; $\mathrm{CAP}_{\mathrm{NGAS}}$, the time of CAP period in NGAS; $\mathrm{CAP}_{\text {sudaslength}}$, the time of CAP period in SUDAS; $\mathrm{CAP}_{\text {sudasslot }}$ number of CAP slots in SUDAS; $d$, the probability of defer transmission that no enough slot is left in the current CAPsudaslength to transmit data frame; $D_{\text {node, }}$ the distance between device nodes and its coordinator; $E_{\text {coord }}$, energy consumption by coordinator node; $E_{\text {dev }}$, energy consumption by device nodes; $E_{\text {total }}$, total energy consumption of the network; GTSlength ${ }_{n}$, the length of GTS allocation for device $n$; GTSstart ${ }_{n}$, the starting time of GTS allocation for device $n$; IDLE, state that a device node has no packet to transmit; $L_{\text {ack }}$, the lengths of ACK is 88 in bits; $L_{\text {beacon }}$ the length of beacon is 760 in bits; $L_{\mathrm{CCA}}$, the lengths of CCA is 32 in bits; $L_{\text {data }}$, the length of data

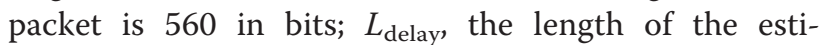
mated remaining CAPsudaslength in bits; $L_{\text {request }}$, the length of GTS request is 72 in bits; $m$, the maximum

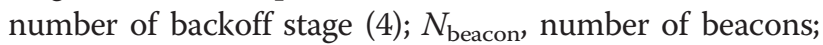
$N_{\text {GTS }}$, number of nodes need to be allocated GTS slot; $N_{\text {GTSrecvcoord }}$ number of GTS packets received by coordinator; $N_{\text {req }}$, number of packets per request; Nsudasslot ${ }_{n}$, number of request slots for each GTS of SUDAS by device $n$; $N_{\text {total }}$, total Number of nodes in the network; $N_{\text {WGTS }}$, number of nodes do not need to be allocated GTS slot;

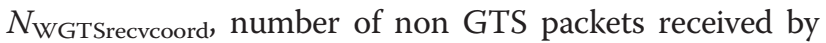
coordinator; $P$, probability that a node generates time critical packets; $P_{\text {CCA1 }}$, steady-state probability for CCA1; $P_{\text {CCA2 }}$, steady-state probability for CCA2; $P_{\text {coll }}$, probability of packet collision in a certain backoff 
stage; $P_{\text {copt }}$, steady-state probability for the collided packet transmission; $P_{\mathrm{cr}}$, probability of a packet being dropped due to collision retransmission; $P_{\text {dropwGTS }}$, probability of non GTS packet dropped as transmitting from device node to its coordinator; $P_{\mathrm{dtx}}$, steady-state probability for waiting time due to defer transmission; $P_{\text {fail1 }}$, probability of fail transmission due to the maximum number of retransmissions after collisions; $P_{\text {fail } 2}$, probability of fail transmission due to no channel to use after reaching the maximum backoff stage at the maximum retransmission stage; $P_{\mathrm{GTS}}$, probability of request GTS allocation; $P_{\text {idle}}$, steady-state probability for idle; $P_{\mathrm{pt}}$, steady-state probability for the packet transmission; $P_{\text {ranb }}$, steady-state probability to perform random backoff; $P_{\mathrm{SG}}$, steady-state probability for successful request GTS packet; $P_{\text {suc }}$, steady-state probability for the successful packet transmission; $P_{\text {sucWGTS, }}$ probability of successful transmission for non GTS packet as transmitting from device node to its coordinator; $P_{\text {tsg, }}$, probability of successful GTS transmis-

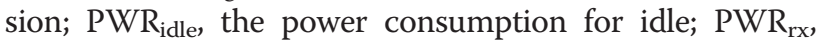
the power consumption for receiving a packet; $\mathrm{PWR}_{\mathrm{tx}}$, the power consumption for transmitting a packet; $R$, the maximum number of retransmissions (3); $r(t)$, number of retransmissions in stochastic state; $R_{\mathrm{b}}$, data rate (250 kbps); $R_{\text {req }}$, GTS request rate of device node; $R_{s}$, symbol rate $(62,500$ symbols/sec); $s(t)$, backoff stage in stochastic state; $S_{\text {CAPsudaslength, the goodput of }}$ $\mathrm{CAP}_{\text {sudaslength }}$ which is a part of CAP in the network; $S_{\text {CFPsudaslength }}$, the goodput of $\mathrm{CFP}_{\text {sudaslength }}$ which is a part of CFP in the network; $\mathrm{SD}_{\text {coord }}$, superframe duration of coordinator; $\mathrm{SO}_{\text {coord }}$, superframe order for coordinator; $S_{\text {total }}$, total goodput of the star network; $T_{\text {ack }}$, time to wait

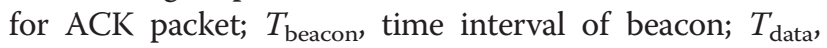
time to transmit data packet; $T_{\text {eCAPsudaslength }}$ the estimated remaining of CAP SUDAS length in time; $T_{\mathrm{f}}$, time of transmit one data packet and receive ACK packet; $T_{\text {Lack}}$, time to transmit ACK packet; $T_{\text {LIFS, }}$ time of IFS duration; $T_{\mathrm{q}}$, time to transmit one packet data and receive ACK packet in CAP SUDAS length; $T_{\text {sd }}$, time of superframe duration; $T_{\text {sim }}$, time of simulation; $T_{\text {slot }}$, time of one slot duration; $T_{\text {sudas }}$, new time of one slot duration in SUDAS; $T_{\mathrm{txCCA}}$, time to transmit CCA; $T x_{n}$, time to transmit data packet according to its arrival rate for device $\mathrm{n}$; $w_{\mathrm{i}}$, backoff window; $X$, probability of collision of packet transmission in a certain backoff stage; $Y$, probability of entering the next backoff stage; $\alpha$, probability that $\mathrm{CCA}_{1}$ is busy; $\alpha_{\text {data }}$, probability that CCA1 is busy due to data packet; $\alpha_{\text {request }}$, probability that CCA1 is busy due to request GTS packet; $\beta$, probability that $\mathrm{CCA}_{2}$ is busy; $\beta_{\text {data }}$, probability that $\mathrm{CCA}_{2}$ is busy due to data packet; $\beta_{\text {request }}$, probability that $\mathrm{CCA}_{2}$ is busy due to request GTS packet; $\lambda_{n}$, arrival rate of data packet for device $\mathrm{n} ; \tau$, probability that a tagged node can transmit a packet; $\phi_{1}$, conditional probability that a tagged node will be at one of the $\mathrm{CCA}_{1}$ states after backoff; $\phi_{2}$, conditional probability that a tagged node will be at one of the $\mathrm{CCA}_{2}$ states after sensing channel idle in the $\mathrm{CCA}_{1}$.

\section{Abbreviations}

ACK: acknowledgement; ANBU: a new GTS allocation scheme with bandwidth utilization; BE: backoff exponent; BI: beacon interval; BO: beacon order; CAP: contention access period; CCA: clear channel assessment; CFP: contention free period; CSMA/CA: carrier sense multiple access with collision avoidance; CW: contention window; GTS: guaranteed time slot; IEEE: Institute of Electrical and Electronics Engineers; IFS: interframe spacing; LIFS: long interframe spacing; LR-WPAN: low-rate wireless personal area network; MAC: medium access control sublayer; NB: number of backoffs; NGAS: a new GTS allocation scheme; PAN: personal area network; PHY: physical layer; SD: superframe duration (in symbols); SIFS: short interframe spacing; SO: superframe order; SUDAS: superframe duration adjustment scheme; UBP: unit backoff period (80 bits).

\section{Competing interests}

The authors declare that they have no competing interests.

\section{Acknowledgements}

This study was supported in part by the Ministry of Science and Technology (MOST) of Taiwan under Grant No. MOST 99-2221-E-011-119.

\section{Author details}

${ }^{1}$ National Taiwan University of Science and Technology, 43, Keelung Rd, Section 4, Taipei 106, Taiwan. ${ }^{2}$ Ling Tung University, 1, Ling Tung Rd, Taichung 408, Taiwan. ${ }^{3}$ Politeknik Elektronika Negeri Surabaya, Kampus ITS Sukolilo, Surabaya 60111, Indonesia.

Received: 11 December 2014 Accepted: 12 February 2015 Published online: 15 April 2015

\section{References}

1. IEEE 802.15.4, part 15.4: wireless medium access control (MAC) and physical layer (PHY) specifications for low-rate wireless personal area networks (WPANs), IEEE standard for information technology. September 2006

2. AN Alvi, SS Naqvi, SH Bouk, N Javaid, U Qasim, ZA Khan, Evaluation of slotted CSMAVCA of IEEE 802.15.4 (Seventh International Conference on Broadband, Wireless Computing, Communication and Applications, Canada, 2012)

3. K Ashrafuzzaman, K Sup Kwak, On the performance analysis of the contention access period of IEEE 802.15.4 MAC. IEEE Commun Lett 15, 9 (2011)

4. T-R Park, T-H Kim, J-Y Choi, S Choi, W-H Kwon, Throughput and energy consumption analysis of IEEE 802.15.4 slotted CSMA/CA. IEEE. Electron Lett 41(18), 1017-1019 (2005)

5. TJ Lee, HR Lee, MY Chung, MAC throughput limit analysis of slotted CSMA/CA in IEEE 802.15.4 WPAN. IEEE Commun Lett 10(7), 561-563 (2006)

6. S Pollin, M Ergen, S Ergen, B Bougard, L Der Perre, I Moerman, A Bahai, P Varaiya, F Catthoor, Performance analysis of slotted carrier sense IEEE 802.15.4 medium access layer. IEEE T Wirel Commun 7(9), 3359-3371 (2008)

7. Y Zhang, F Shu, Packet Size Optimization for Goodput and Energy Efficiency Enhancement in Slotted IEEE 802.15.4 Networks. IEEE Wireless Communications and Networking Conference, 2009, pp. 1-6

8. J He, Z Tang, HH Chen, Q Zhang, An accurate and scalable analytical model for IEEE 802.15.4 slotted CSMA/CA networks. IEEE T Wirel Commun 8(1), 440-448 (2009)

9. Z Xiao, C He, L Jiang, An analytical model for IEEE 802.15.4 with sleep mode based on time-varying queue, in IEEE International Conference on Communications (ICC), Kyoto, Japan, 2011

10. C Buratti, Performance analysis of IEEE 802.15 .4 beacon-enabled mode. IEEE Trans. Veh. Technol 59, 2031-2045 (2010)

11. Z Tao, S Panwar, D Gu, J Zhang, Performance analysis and a proposed improvement for the IEEE 802.15.4 contention access period. IEEE WCNC 4, 1811-1818 (2006)

12. P Park, P Di Marco, P Soldati, C Fischione, KH Johansson, A Generalized Markov Chain Model for Effective Analysis of Slotted IEEE 802.15.4. IEEE 6th International Conference on Mobile Adhoc and Sensor Systems, 2009, pp. 130-139 
13. YK Huang, AC Pang, HN Hung, A comprehensive analysis of low-power operation for beacon-enabled IEEE 802.15.4 wireless networks. IEEE T Wirel Commun 8(11), 5601-5611 (2009)

14. M Khanafer, M Guennoun, HT Mouftah, Adaptive Sleeping Periods in IEEE 802.15.4 for Efficient Energy Savings: Markov-based Theoretical Analysis, in IEEE International Conference on Communications (ICC), Kyoto, Japan, 2011

15. B Gao, C He, L Jiang, Modeling and Analysis of IEEE 802.15.4 CSMAVCA with Sleep Mode Enabled. International Conference on Communication Systems, pp.6-11, Guangzhou, China, 2008

16. C-Y Jung, H-Y Hwang, D-K Sung, G-U Hwang, Enhanced Markov chain model and throughput analysis of the slotted CSMAVCA for IEEE 802.15.4 under unsaturated traffic conditions. IEEE T Veh Technol 58(1), 473-478 (2009)

17. B Shrestha, E Hossain, S Camorlinga, A Markov model for IEEE 802.15.4 MAC with GTS transmissions and heterogeneous traffic in non-saturation mode, in IEEE International Conference on Communication Systems (ICCS), pp. 56-61, Singapore, 2010

18. $\mathrm{B}-\mathrm{H}$ Lee, M Udin Harun Al Rasyid, $\mathrm{H}-\mathrm{K}$ Wu, Analysis of Superframe Adjustment and Beacon Transmission for IEEE 802.15.4 Cluster Tree Networks. EURASIP Journal on Wireless Communication and Networking, July 2012

19. M Martalo, S Busanelli, G Ferrari, Markov Chain-based performance analysis of multihop IEEE 802.15.4 wireless networks. Perform Evaluation 66, 722-741 (2009)

20. P Park, C Fischione, K Johansson, Performance analysis of GTS allocation in beacon enabled IEEE 802.15.4, in $6^{\text {th }}$ Annual IEEE Communications Society Conference on Sensor, Mesh and Ad Hoc Communications and Networks, 2009, pp. 1-9

21. A Koubaa, M Alves, E Tovar, GTS allocation analysis in IEEE 802.15 .4 for real-time wireless sensor networks. Paper presented at the 20th International Parallel and Distributed Processing Symposium, IEEE, Rhodes Island, 25-29 April 2006

22. S-T Sheu, YY Shih, WT Lee, CSMA/CF Protocol for IEEE 802.15.4 WPANs. IEEE Trans. Veh. Technol. 58(3), 1501-1516 (2009)

23. L-C Ko, Z-T Chou, A Novel Multi-Beacon Superframe Structure with Greedy GTS Allocation for IEEE 802.15.4 Wireless PANs. IEEE Wireless Communications and Networking Conference (WCNC), pp.2328-2333, Kowloon, March, 2007.

24. A Koubaa, M Alves, E Tovar, A Cunha, An implicit GTS allocation mechanism in IEEE 802.15.4 for time-sensitive wireless sensor networks: theory and practice. Springer Real-Time Systems 39, 169-204 (2008)

25. H-W Cho, S-J Bae, M-Y Chung, Utilization-aware dynamic GTS allocation scheme in IEEE 802.15.4. 010 16th Asia-Pacific Conference on Communications (APCC), 2010, pp. 210-214

26. Y-K Huang, A-C Pang, H-N Hung, An adaptive GTS allocation scheme for IEEE 802.15.4. IEEE T Parall Distr 19(5), 641-651 (2008)

27. L Cheng, AG Bourgeois, X Zhang, A new GTS allocation scheme for IEEE 802.15.4 networks with improved bandwidth utilization. Communications and Information Technologies, 2007. ISCIT '07. International Symposium, 2007, pp. $1143-1148$

28. B Shrestha, E Hossain, S Camorlinga, R Krishnamoorthy, D Niyato, An Optimization-Based GTS Allocation Scheme for IEEE 802.15.4 MAC with Application to Wireless Body-Area Sensor Networks. 2010 IEEE International Conference on Communications (ICC), 2010, pp. 1-6

29. L Yang, S Zeng, A New GTS Allocation Schemes for IEEE 802.15.4. 2012 5th International Conference on BioMedical Engineering and Informatics, 2012 pp. 1398-1401

30. YS Seo, DY Kim, J Cho, A dynamic CFP allocation and opportunity contention-based WBAN MAC protocol. IEEE T Wirel Commun E93.B(4), 850-853 (2010)

31. H-K Wu, B-H Lee, M Udin Harun Al Rasyid, Study on Superframe Adjustment for Cluster Tree in Wireless Sensor Networks. Proceedings of the 2012 IEEE International Conference on Cyber Technology in Automation, Control and Intelligent Systems, 2012, pp. 43-47

32. AS Chipcon SmartRF ${ }^{\circledR}$ CC2420 datasheet (rev 1.2), Chipcon corp., 2004.

33. B Bougard, F Catthoor, DC Daly, A Chandrakasan, W Dehaene, Energy Efficiency of the IEEE 802.15.4 Standard in Dense Wireless Microsensor Networks: Modeling and Improvement Perspectives. Proceeding of Design, Automation and Test in Europe Conference and Exhibition. (DATE'05), pp.196-201, Mar. 2005

\section{Submit your manuscript to a SpringerOpen ${ }^{\circ}$ journal and benefit from:}

- Convenient online submission

- Rigorous peer review

- Immediate publication on acceptance

- Open access: articles freely available online

- High visibility within the field

- Retaining the copyright to your article

Submit your next manuscript at $>$ springeropen.com 